\title{
Prediction-Based Stabilization of Linear Systems Subject to Input-Dependent Input Delay of Integral-Type
}

\author{
Delphine Bresch-Pietri, Jonathan Chauvin, and Nicolas Petit
}

\begin{abstract}
In this paper, it is proved that a predictor-based feedback controller can effectively yield asymptotic convergence for a class of linear systems subject to input-dependent input delay. This class is characterized by the delay being implicitly related to past values of the input via an integral model. This situation is representative of systems where transport phenomena take place, as is frequent in the process industry. The sufficient conditions obtained for asymptotic stabilization bring a local result and require the magnitude of the feedback gain to be consistent with the initial conditions scale. Arguments of proof for this novel result include general Halanay inequalities for delay differential equations and build on recent advances of backstepping techniques for uncertain or varying delay systems.
\end{abstract}

Index Terms-Backstepping, delay differential equation, partial differential equation, prediction-based feedback, time-delay systems.

\section{Introduction And PRoblem Statement}

$\mathbf{N}$ UMEROUS control systems involve a physical lag which, as it is well known, reveals troublesome in the design and tuning of feedback control laws. In practice, such dead-time can occur when sensors and actuators are not co-located. Prime examples are processes involving transportation of material, such as mixing processes for liquid or gaseous fluids, chemical reactors [16], automotive engine [17] and exhaust line [13], heat collector plant [32], blending in liquid or solid networks [12], crushing mill [31] and batch processes [30], to name a few. Remarkably, in all these examples, the lag directly depends on the control variable and the considered delay is inherently input-dependent.

Surprisingly, it seems that stabilization of such processes with input-dependent delay in the input $D(u)$ or $D\left(u_{t}\right)$, where $u_{t}$ denotes past values over a finite horizon, has seldom been theoretically studied. Rather, a widely considered approach is to neglect to various extents the control-dependency of the delay,

Manuscript received June 18, 2013; revised December 4, 2013; accepted April 15, 2014. Date of publication May 6, 2014; date of current version August 20, 2014. Recommended by Associate Editor P. Pepe.

D. Bresch-Pietri is with the Department of Mechanical Engineering at Massachusetts Institute of Technology, Cambridge MA 02139, USA (e-mail: dbp@mit.edu).

J. Chauvin is with the Département Contrôle, Signal et Système at IFP Energies nouvelles, Rueil Malmaison 92852, France.

N. Petit is with the Centre Automatique et Systèmes at MINES ParisTech, 75272, Paris Cedex 06, France.

Color versions of one or more of the figures in this paper are available online at http://ieeexplore.ieee.org.

Digital Object Identifier 10.1109/TAC.2014.2322238 e.g., by modeling this dependence as $D(u) \approx D(t)$ (or even by a constant average value $D$ ) and by asking the controller to deal with a certain level of unstructured delay uncertainty.

On the opposite, the methodology developed in this paper explicitly accounts for this dependency with the objective of improving transient performances. The scope of this method is as follows: we investigate the following potentially unstable Single-Input Single-Output linear time-invariant plant, ${ }^{1}$ with a measured output $x(t)$

$$
x^{(n)}+a_{n-1} x^{(n-1)}+\ldots+a_{1} \dot{x}+a_{0} x=b_{0} \varphi(t-D(t))
$$

driven by a delayed input, where the varying delay $D(t)$ is implicitly defined in terms of the input past values as

$$
\int_{t-D(t)}^{t} \varphi(s) d s=1 \text { with } \varphi(t)=\operatorname{Sat}_{[\underline{u},+\infty)}(u(t)), \quad \underline{u}>0 .
$$

This system is depicted in Fig. 1. The integral delay model ${ }^{2}$ (2) corresponds to a Plug-Flow assumption [29] in a transport phenomenon and encompasses of a wide class of systems involving transport phenomena with velocity $\varphi$ [7], [38]. It can be understood as the time of propagation for a transport process with time-varying speed for which the control variable is the speed itself. ${ }^{3}$

Motivating Example: An example of system fitting inside this framework is the one of the exhaust Fuel/Air Ratio (FAR) for Gasoline engines which can be found, e.g., in [7]. A schematic view of the system is pictured in Fig. 2. To optimize the catalyst operation, one wants to regulate the exhaust mixture composition $\lambda$ to stoichiometry. This is done by injecting a given amount of fuel inside the combustion chamber, according to the current fresh air mass flow rate. However, such a variable fluctuates with the driver torque request (e.g., tip-ins and tip-outs). The main challenge while trying to improve the

\footnotetext{
${ }^{1}$ Potential existing zeros can still be handled by a suitable aforementioned choice of state-space representation and of the output matrix.

${ }^{2}$ Because $\varphi \geq \underline{u}>0$, this transport delay is well-defined (positive and timederivative bounded by one) and upper-bounded, $D(t) \leq \underline{D} \triangleq(1 / \underline{u}) t \geq 0$. Further, as $D \mapsto \int_{t-D}^{t} \varphi(s) d s$ is strictly increasing, it is invertible and the delay can be calculated. It is therefore considered as known in this paper.

${ }^{3}$ In details, it can be shown that the solution of the transport PDE $\partial_{t} \xi(x, t)=$ $u(t) \partial_{x} \xi(x, t)$ for $x=[0,1]$ satisfies $\xi(1, t)=\xi(0, t-D(t))$ with $D(t)$ defined by (2) (see [7]).
} 


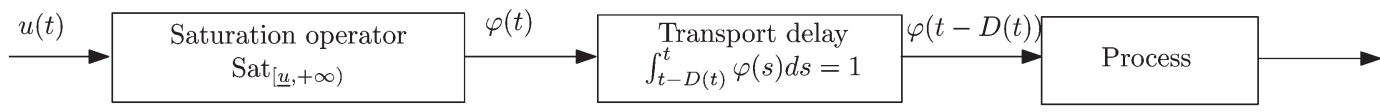

Fig. 1. The addressed problem, where the input is delayed by a transport delay which is input-varying.



Fig. 2. Schematic view of the Fuel/Air Ratio system on a Gasoline engine equipped with indirect injection. A given amount of fuel is provided by the injector (actuator). The control objective here is to guarantee that the exhaust Fuel/Air Ratio, downstream of the catalyst (measured by an oxygen sensor), is close to stoichiometry.

transient responses corresponding to these stimuli is that the injector (actuator) is located typically upstream of the combustion chamber. It is therefore spatially distant from the dynamics under consideration. This generates a transport delay (see [36]) which follows an integral relation of type (2).

In details, considering a given operating point (constant fresh air mass flow rate), defining $u(t)$ as the injected fuel flow rate and $x(t)$ as the exhaust FAR, which is the measured output, the dynamics can be written as a (stable) second-order input delay system (see [7] for specific modeling). The transport delay bearing on the input varies with the exhaust gas flow rate, which itself varies with the exhaust pressure [11]. The latter is a function of the injected fuel flow rate. Linearizing around the given operating point (potentially after normalization), one can recover an integral relation of type (2). Further, the input is lower-saturated to prevent the engine to stall (a minimum amount of fuel $\underline{u}$ has to be injected for the mixture in the chamber to burn), which justifies the saturation in (2). Finally, we wish to stabilize the plant toward a positive equilibrium (stoichiometric exhaust composition) corresponding to a control reference $u^{r}>0$.

Proposed Methodology and Comparison With State-of-theArt Techniques: The control objective at stake in this paper is to stabilize the plant (1), (2) over any equilibrium point such that $x^{r}=\left(b_{0} / a_{0}\right) u^{r}$ with $u^{r} \geq \underline{u}>0$. The reason why we only consider strictly positive steady-states is that, following (2), $D$ tends to infinity while $\varphi$ tends to zero. Therefore, in this case, the inputs do not reach the plant anymore and, obviously, unstable systems cannot be stabilized.

The solution we consider is a predictor-based control strategy (straightforwardly generalizing the ideas of [1], [24], [33]). Prediction is already widely used for systems with constant input time-delays (see for instance [8], [14], [19], [25], [26] or [31] and the references therein) but is still not of general use for time-varying delays (see [28] or, more recently, [22]). In such cases, to compensate the varying input delay, the prediction has to be calculated over a time window of which length matches the value of the future delay. In other words, one needs to predict the future variations of the delay to compensate it. For example, this is the approach followed in [35] for a communication time-varying delay, the variations of which are provided by a given known model. It has also been used in [3], [4] for a state-dependent delay or in [5] for a delay depending on delayed state, where variations are anticipated by a careful prediction of the system state.

When the delay depends on the input, things are getting very involved. Determining the required prediction horizon becomes an implicit question, which may not be practically solvable nor even well-posed (this implicit interaction may yield to a set of conditions characterizing the time horizon which may be unsolvable for certain initial conditions). This implicit nature is caused by the reciprocal interactions between the control (current and past) values and the delay, yielding a closed-loop dependency.

For this reason, in this paper, in lieu of seeking exact delay compensation, we consider a prediction horizon equal to the current delay value. This does not prevent implicit mutual dependency between delay and input from arising, as the delay model (2) is input-varying and the delay is involved in the prediction-based control law. So, to disrupt this loop, which is particularly troublesome in the stability analysis, we use a twosteps methodology.

In a first move (Section II of this paper), we consider the input-dependency as a particular type of time-variations. This virtual parametrization suggests us to derive a robust compensation result for linear systems with time-varying input delay, using the backstepping tools proposed in [21] for the analysis of input-delay systems stability. Technically, this result guarantees stabilization provided that the delay variations are sufficiently small. This rather reasonable condition can be easily interpreted as a direct consequence of the approximation of the prediction interval by the current delay value. In a second step (Section III of this paper), to derive a more practical sufficient condition, the delay variations are related to the control tracking error fluctuations, which are analyzed using delay differential equations (DDE) stability theory (Halanay-type inequalities [15]). This methodology allows us to establish a result guaranteeing local exponential stability, i.e., Theorem 1, which yields exponential convergence of the system output towards $x^{r}$ provided the initial conditions ( $x$, its derivatives and the past values of the control $\left.u_{[-D(0), 0]}\right)$ are sufficiently close to the equilibrium.

To the best of the authors' knowledge, this connection between prediction-based control law and input-dependency of the delay has not been studied earlier, except on preliminary works by the authors ([9] in which a scalar system is studied 
and [10] in which state-feedback is considered). The output prediction-based feedback proposed here for potentially unstable systems of order $n$ subject the input-dependent delay (2), along with its proof of convergence in Theorem 1, is the main contribution of this paper.

The paper is organized as follows. In Section II, we address the topic of robust compensation of time-varying delay. Then, sufficient conditions for stabilization in the case of input-varying delay are derived in Section III through a proof of convergence invoking Halanay-like inequalities arguments. Finally, the merits of the obtained result are then illustrated in Section IV through simulations.

Notations and Definitions: In the following, |.| stands for the usual Euclidean norm, $\|f(t)\|$ represents the norm of a spatially distributed variable $f:(x, t) \in[0,1] \times \mathbb{R} \mapsto \mathbb{R}$ and is defined as

$$
\|f(t)\|=\sqrt{\int_{0}^{1} f(x, t)^{2} d x}
$$

$\mathcal{C}^{0}\left(S_{1}, S_{2}\right)$ (resp. $\left.\mathcal{C}^{1}\left(S_{1}, S_{2}\right)\right)$ denotes the set of continuous (resp. continuously differentiable) functions defined on a set $S_{1}$ with values into a set $S_{2}$. The operator Sat ${ }_{I}$ stands for the standard saturation operator onto the interval $I$. For a given symmetric matrix, $\underline{\lambda}($.$) and \bar{\lambda}($.$) stand respectively for$ minimum and maximum eigenvalues.

We write $\partial_{x} f$ the partial derivative of a function $\mathrm{f}$ with respect to a variable $x . x_{t}$ refers to the function $x_{t}: s \in[-\underline{D}, 0] \mapsto$ $x(t+s)$ for a given function $x$ and a constant $\underline{D}>0$. For any continuous function $k$ defined on $[-\underline{D}, 0]$ and a polynomial function $\pi$, we write $\pi\left(x_{t}\right)=\pi\left(x\left(t_{1}\right), \ldots, x\left(t_{n-2}\right)\right.$, $\left.\int_{t_{n-1}}^{t_{n}} k(t-s) x(s) d s\right)$ for $\left(t_{1}, \ldots, t_{n}\right) \in[t-\underline{D}, t]^{n}$.

A polynomial function $\pi$ in the variables $\left(x_{1}, \ldots, x_{m}, \ldots\right.$, $x_{n}$ ) is said to be at least quadratic in $x_{1}, \ldots, x_{m}$ iff, for any given $\left(x_{m+1}, \ldots, x_{n}\right)$, the corresponding polynomial function $\pi_{m}$ defined as

$$
\pi_{m}\left(x_{1}, \ldots, x_{m}\right)=\pi\left(x_{1}, \ldots, x_{m}, \ldots, x_{n}\right)
$$

has no terms of order 0 or 1, e.g., both $\pi=x_{1}^{2}+x_{1} x_{2} x_{3}$ and $\pi=x_{2} x_{1}+x_{3} x_{1}^{2}$ are at least quadratic in $\left(x_{1}, x_{2}\right)$ while this is not the case for $\pi=x_{3}+x_{3} x_{2}^{2}$.

\section{SufFicient CONDITION For Robust COMPENSATION OF A TIME-VARYING INPUT DELAY}

In this section, we consider the input delay as being any element of $\mathcal{C}^{1}\left(\mathbb{R}_{+},[0, \underline{D}]\right)$ for some $\underline{D}$. Here, to design our controller, we do not relate the variations of $D(t)$ to those of the control variable. To use the stabilization result established below, we first reformulate system (1), (2) under a state-space representation

$$
\left\{\begin{array}{l}
\dot{X}=A X(t)+B \varphi(t-D(t)) \\
Y(t)=C X(t) \\
\int_{t-D(t)}^{t} \varphi(s) d s=1 \quad \text { with } \varphi(t)=\operatorname{Sat}_{[\underline{u},+\infty)}(u(t))
\end{array}\right.
$$

where the pair $(A, C)$ is observable and the controllable pair $(A, B)$ is

$$
A=\left(\begin{array}{cccc}
0 & 1 & & 0 \\
\vdots & & \ddots & \\
0 & 0 & & 1 \\
-a_{0} & -a_{1} & \ldots & -a_{n-1}
\end{array}\right), \quad B=\left(\begin{array}{c}
0 \\
\vdots \\
0 \\
b_{0}
\end{array}\right) .
$$

Lemma 1: Consider the closed-loop single input system

$$
\begin{aligned}
& \dot{X}(t)=A X(t)+B u(t-D(t)) \\
& Y(t)=C X(t) \\
& \dot{\hat{X}}(t)=A \hat{X}(t)+B u(t-D(t))-L[Y(t)-C \hat{X}(t)] \\
& u(t)=K\left[e^{A D(t)} \hat{X}(t)+\int_{t-D(t)}^{t} e^{A(t-s)} B u(s) d s\right]
\end{aligned}
$$

where $X \in \mathbb{R}^{n}, u \in \mathbb{R}, K$ and $L$ are chosen such that, respectively, $A+B K$ and $A+L C$ are Hurwitz and $D \in$ $\mathcal{C}^{1}\left(\mathbb{R}_{+},[0, \underline{D}]\right)$ for some $\underline{D}>0$. Define

$$
\begin{gathered}
\Upsilon_{0}(t)=|X(t)|^{2}+|X(t)-\hat{X}(t)|^{2}+\int_{t-D(t)}^{t} u(s)^{2} d s \\
+D(t)^{2} \int_{t-D(t)}^{t} \dot{u}(s)^{2} d s
\end{gathered}
$$

There exists $\Delta^{*}(K) \in(0,1)$ such that, if

$$
\forall t \geq 0, \quad|\dot{D}(t)|<\Delta^{*}(K)
$$

then there exist $R, \rho>0$ such that

$$
\forall t \geq 0, \quad \Upsilon_{0}(t) \leq R \Upsilon_{0}(0) e^{-\rho t}
$$

and in particular the system state $X$ of plant (7a) exponentially converges to the origin.

The prediction controller (7d) is a direct extension of the prediction over a constant delay time window to time-varying delay and output feedback, replacing respectively the constant delay by $D(t)$ and the system state by its observer $\hat{X}$. This controller aims at forecasting values of the state over a time window of varying length $D(t){ }^{4}$ Of course, exact

\footnotetext{
${ }^{4}$ Note that, even if $\hat{X}=X$, this controller does not exactly match the predicted system state on a time-horizon $D(t)$. Indeed, using the variation of constant formula

$\forall t \geq 0, \quad X(t+D(t))=e^{A D(t)} X(t)$

$$
+\int_{t-D(t)}^{t} e^{A(t-s)} B u(s+D(t)-D(s)) d s .
$$

Under the assumption that the variations of the delay are sufficiently small, this latter integral can be approximated by the one used in (7d) as $D(t)-D(s) \approx$ 0 . As this assumption is already required to robustly compensate the delay, we rather use the prediction form (7d) which is easier to implement instead of the true prediction given above.
} 
compensation of the delay is not achieved with this controller ${ }^{5}$ and can be highly inaccurate when the delay is fast varying. In this context, (9) can be interpreted as a condition for robust delay compensation achievement. ${ }^{6}$ The spirit of this condition is that, if the delay varies sufficiently slowly, its current value $D(t)$ used for prediction remains close enough to its future values, and the corresponding prediction is accurate enough to guarantee the stabilization of the plant through the feedback loop.

We now detail the proof of this result.

Proof: In the following, we use the Lyapunov tools introduced in [21] to analyze the stability of input time-delay systems and which are based on a backstepping transformation of a certain actuator state defined for constant delays. First, we extend this distributed input to handle time-varying delay by defining $v(x, t)=u(t+D(t)(x-1)) x \in[0,1]$ which enables to rewrite plant $(7 \mathrm{a})-(7 \mathrm{c})$ as

$$
\left\{\begin{array}{l}
\dot{\tilde{X}}(t)=(A+L C) \tilde{X}(t) \\
\dot{\hat{X}}(t)=A \hat{X}(t)+B v(0, t)-L C \tilde{X}(t) \\
D(t) \partial_{t} v(x, t)=\partial_{x} v(x, t)+\dot{D}(t)(x-1) \partial_{x} v(x, t) \\
v(1, t)=u(t)
\end{array}\right.
$$

in which the state observation error $\tilde{X}=X-\hat{X}$ has been introduced. In details, the input delay is now represented as a cascade with a transport partial differential equation (PDE) driven by the input and where the convection speed varies both with space and time. Pursuing the mentioned approach, now, consider the following backstepping transformation of the distributed input

$$
\begin{array}{r}
w(x, t)=v(x, t)-D(t) \int_{0}^{x} K e^{A D(t)(x-y)} B v(y, t) d y \\
-K e^{A D(t) x} \hat{X}(t)
\end{array}
$$

which is designed to satisfy $w(1, t)=0$ compliantly with the choice of the control law (7d). The error (11) can now be rewritten as

$$
\left\{\begin{array}{l}
\dot{\tilde{X}}(t)=(A+L C) \tilde{X}(t) \\
\dot{\hat{X}}(t)=(A+B K) \hat{X}(t)+B w(0, t)-L C \tilde{X}(t) \\
D(t) \partial_{t} w=\partial_{x} w+\dot{D}(t) g(x, t)+D K e^{A D(t) x} L C \tilde{X}(t) \\
w(1, t)=0
\end{array}\right.
$$

\footnotetext{
${ }^{5}$ As discussed earlier, to do so, one would need to consider a time window of which length would exactly match the value of the future delay, as it is made in [28] and [22]. In details, defining $\eta(t)=t-D(t)$ and assuming that its inverse exists (which is the case if $\dot{D}<1$ ), exact delay-compensation is obtained with the feedback law $U(t)=K X\left(\eta^{-1}(t)\right)$. Yet, implementing this relation requires to predict the future variation of the delay via $\eta^{-1}(t)$, which may not practically achievable for an input-varying delay.

${ }^{6}$ Interestingly, a similar condition is often stated in Linear Matrix Inequality approaches, such as [37] for example, where the delay is also assumed to be time-differentiable.
}

with

$$
\begin{array}{r}
g(x, t)=(x-1) \partial_{x} v(x, t)-K D(t) \int_{0}^{x} e^{A D(t)(x-y)} B(y-1) \\
\times \partial_{x} v(y, t) d y-K D(t)\left[\int_{0}^{x} e^{A D(t)(x-y)}(I+A D(t)(x-y))\right. \\
\left.\times B v(y, t) d y+K A x e^{A D(t) x} \hat{X}(t)\right]
\end{array}
$$

which can be formulated in terms of $\tilde{X}, \hat{X}, w$ and $w_{x}$ using the inverse Volterra equation corresponding to (12). For the Lyapunov analysis below, we also need the governing equation of the spatial derivative of this transformed distributed actuator which is

$$
\left\{\begin{array}{l}
D(t) \partial_{x t} w=\partial_{x x} w+\dot{D}(t) \partial_{x} g(x, t)+D^{2} K e^{A D(t) x} A L C \tilde{X}(t) \\
\partial_{x} w(1, t)=-\dot{D}(t) g(1, t)-D(t) K e^{A D(t)} L C \tilde{X}(t)
\end{array}\right.
$$

We can now start the Lyapunov analysis and introduce the following Lyapunov-Krasovskii functional candidate

$$
\begin{aligned}
V(t)= & \tilde{X}(t)^{T} P_{0} \tilde{X}(t)+b_{0} \hat{X}(t)^{T} P_{1} \hat{X}(t) \\
& +b_{1} D(t) \int_{0}^{1}(1+x)\left[w(x, t)^{2}+\left(\partial_{x} w(x, t)\right)^{2}\right] d x
\end{aligned}
$$

where $P_{0}$ and $P_{1}$ are the symmetric definite solutions of the two Lyapunov equations $(A+L C)^{T} P_{0}+P_{0}(A+L C)=$ $-Q_{0}$ and $(A+B K)^{T} P_{1}+P_{1}(A+B K)=-Q_{1}$ for two given symmetric definite positive matrices $Q_{0}$ and $Q_{1}$. Taking a time derivative of this functional and using integration by parts yield

$$
\begin{aligned}
\dot{V}(t)= & -\tilde{X}(t)^{T} Q_{0} \tilde{X}(t)-b_{0} \hat{X}(t)^{T} Q_{1} \hat{X}(t)-b_{1} w(0, t)^{2} \\
& +2 b_{0} \hat{X}(t)^{T} P_{1} B w(0, t)-2 b_{0} \hat{X}(t)^{T} P_{1} L C \tilde{X}(t) \\
& -b_{1}\|w(t)\|^{2}+2 b_{1}\left(\partial_{x} w(1, t)\right)^{2}-b_{1}\left(\partial_{x} w(0, t)\right)^{2} \\
& -b_{1}\left\|\partial_{x} w(t)\right\|^{2}+2 b_{1} \dot{D}(t) \int_{0}^{1}(1+x) w(x, t) g(x, t) d x \\
& +2 b_{1} \int_{0}^{1}(1+x) w(x, t) D(t) K e^{A D(t) x} L C \tilde{X}(t) d x \\
& +2 b_{1} \dot{D}(t) \int_{0}^{1}(1+x) \partial_{x} w(x, t) \partial_{x} g(x, t) d x
\end{aligned}
$$




$$
\begin{aligned}
& +b_{1} \dot{D}(t) \int_{0}^{1}(1+x)\left[w(x, t)^{2}+\left(\partial_{x} w(x, t)\right)^{2}\right] d x \\
& +2 b_{1} \int_{0}^{1}(1+x) \partial_{x} w(x, t) D(t)^{2} K e^{A D(t) x} A L C \tilde{X}(t) d x .
\end{aligned}
$$

To bound the remaining terms, one can introduce the inverse transformation of (12)

$$
\begin{array}{r}
v(x, t)=w(x, t)+D(t) K \int_{0}^{x} e^{(A+B K) D(t)(x-y)} B w(y, t) d y \\
+K e^{(A+B K) D(t) x} \tilde{X}
\end{array}
$$

and its spatial derivative to obtain, using Cauchy-Schwartz's and Young's inequality, the existence of positive constants $M_{1}$, $M_{2}, M_{3}$ and $M_{4}$ such that

$$
\begin{aligned}
& \left|2 \int_{0}^{1}(1+x) w(x, t) g(x, t) d x\right| \\
& \leq M_{1}\left(|\hat{X}(t)|^{2}+\|w(t)\|^{2}+\left\|w_{x}(t)\right\|^{2}\right) \\
& \left|2 \int_{0}^{1}(1+x) w(x, t) D(t) K e^{A D(t) x} L C \tilde{X}(t) d x\right| \\
& \leq M_{2}|\tilde{X}(t)|^{2}+\frac{\|w(t)\|^{2}}{2} \\
& \left|2 \int_{0}^{1}(1+x) \partial_{x} w(x, t) \partial_{x} g(x, t) d x\right| \\
& \leq M_{3}\left(|\hat{X}(t)|^{2}+\|w(t)\|^{2}+\left\|\partial_{x} w(t)\right\|^{2}+\left(\partial_{x} w(0, t)\right)^{2}\right)
\end{aligned}
$$

$$
\begin{aligned}
& \left|2 \int_{0}^{1}(1+x) \partial_{x} w(x, t) D(t)^{2} K e^{A D(t) x} A L C \tilde{X}(t) d x\right| \\
& \quad \leq M_{4}|\tilde{X}(t)|^{2}+\frac{\left\|\partial_{x} w(t)\right\|^{2}}{2} \\
& 2\left(\partial_{x} w(1, t)\right)^{2} \leq M_{6}|\tilde{X}(t)|^{2} \\
& \quad+M_{5}|\dot{D}(t)|^{2}\left(|\hat{X}(t)|^{2}+\|w(t)\|^{2}+\left\|\partial_{x} w(t)\right\|^{2}\right) .
\end{aligned}
$$

Further, using Young's inequality, we have that

$$
\begin{aligned}
& \left|2 b_{0} \hat{X}(t)^{T} P_{1} B w(0, t)\right| \\
& \quad \leq \frac{b_{0} \underline{\lambda}\left(Q_{1}\right)}{4}|\hat{X}(t)|^{2}+\frac{4 b_{0}}{\underline{\lambda}\left(Q_{1}\right)}\left|P_{1} B\right|^{2} w(0, t)^{2} \\
& \left|2 b_{0} \hat{X}(t)^{T} P_{1} L C \tilde{X}(t)\right| \\
& \quad \leq \frac{b_{0} \underline{\lambda}\left(Q_{1}\right)}{4}|\hat{X}(t)|^{2}+\frac{4 b_{0}}{\underline{\lambda}\left(Q_{1}\right)}\left|P_{1} L C\right|^{2}|\tilde{X}(t)|^{2} .
\end{aligned}
$$

Therefore, defining the intermediate functional $V_{0}=|\tilde{X}(t)|^{2}+$ $|\hat{X}(t)|^{2}+\|w(t)\|^{2}+\left\|\partial_{x} w(t)\right\|^{2}$, using (19)-(25) and regrouping terms, it is straightforward to get

$$
\begin{gathered}
\dot{V}(t) \leq-\frac{b_{0} \underline{\lambda}\left(Q_{1}\right)}{2}|\hat{X}(t)|^{2}-\left(b_{1}-4 \frac{b_{0}}{\underline{\lambda}\left(Q_{1}\right)}\left|P_{1} B\right|^{2}\right) w(0, t)^{2} \\
-\left(\underline{\lambda}\left(Q_{0}\right)-4 \frac{b_{0}}{\underline{\lambda}\left(Q_{1}\right)}\left|P_{1} L C\right|^{2}-b_{1}\left(M_{2}+M_{6}+M_{4}\right)\right)|\tilde{X}(t)|^{2} \\
-\frac{b_{1}}{2}\|w(t)\|^{2}-\frac{b_{1}}{2}\left\|\partial_{x} w(t)\right\|^{2}-b_{1}\left(1-|\dot{D}(t)| M_{3}\right)\left(\partial_{x} w(0, t)\right)^{2} \\
+|\dot{D}(t)| b_{1}\left(M_{1}+M_{5}|\dot{D}(t)|+M_{3}+2\right) V_{0} .
\end{gathered}
$$

Consequently, by choosing $b_{1}=\left(8 b_{0} / \underline{\lambda}\left(Q_{1}\right)\right)\left|P_{1} B\right|^{2}$ and $b_{0}=$ $\left(\underline{\lambda}\left(Q_{0}\right) \underline{\lambda}\left(Q_{1}\right) / 8\left(\left|P_{1} L C\right|^{2}+2\left(M_{2}+M_{4}+M_{6}\right)\left|P_{1} B\right|^{2}\right)\right)$, one can define

$$
\begin{aligned}
\eta & =\min \left\{\frac{\lambda\left(Q_{0}\right)}{2}, \frac{b_{0} \underline{\lambda}(Q 1)}{2}, \frac{b_{1}}{2}\right\} \\
\Delta^{*}(K) & =\min \left\{1, \frac{1}{M_{3}}, \frac{\eta}{b_{1}\left(M_{1}+M_{5}+M_{3}+2\right)}\right\}
\end{aligned}
$$

and obtain that, if $|\dot{D}(t)|<\Delta^{*}(K), t \geq 0$ then there exists $\mu>$ 0 such that

$$
\begin{aligned}
\dot{V}(t) & \leq-\mu V_{0}(t) \\
& \leq-\frac{\mu}{\max \left\{\bar{\lambda}\left(P_{0}\right), b_{0} \bar{\lambda}\left(P_{1}\right), 2 b_{1} \bar{D}\right\}} V(t) \\
& =-\mu_{1} V(t)
\end{aligned}
$$

in which $\mu_{1}$ is a positive constant. Consequently

$$
\forall t \geq 0, \quad V(t) \leq V(0) e^{-\mu_{1} t} .
$$

Finally, one can reformulate this result in terms of the functional $\Upsilon_{0}$. Indeed, using a simple change of variable, $\Upsilon_{0}$ can be rewritten as

$$
\Upsilon_{0}(t)=|X(t)|^{2}+|\tilde{X}(t)|^{2}+D(t)\|v(t)\|^{2}+D(t)\left\|\partial_{x} v(t)\right\|^{2} .
$$

Then, using (18) together with Young's inequality, one can establish the following inequalities:

$$
\begin{gathered}
\|v(t)\|^{2} \leq r_{1}|\hat{X}(t)|^{2}+r_{2}\|w(t)\|^{2} \\
\left\|\partial_{x} v(t)\right\|^{2} \leq r_{3}|\hat{X}(t)|^{2}+r_{4}\|w(t)\|^{2}+r_{5}\left\|\partial_{x} w(t)\right\|^{2} \\
\|w(t)\|^{2} \leq s_{1}|\hat{X}(t)|^{2}+s_{2}\|v(t)\|^{2} \\
\left\|\partial_{x} w(t)\right\|^{2} \leq s_{3}|\hat{X}(t)|^{2}+s_{4}\|v(t)\|^{2}+s_{5}\left\|\partial_{x} v(t)\right\|^{2}
\end{gathered}
$$

where $r_{1}, r_{2}, r_{3}, r_{4}, r_{5}, s_{1}, s_{2}, s_{3}, s_{4}$ and $s_{5}$ are positive constants. Therefore, using (32)-(35), one can obtain, for $t \geq 0$

$$
\begin{aligned}
& \frac{1}{\max \left\{\bar{\lambda}\left(P_{0}\right)+b_{0} \bar{\lambda}\left(P_{1}\right), 2 b_{1}\right\}} V(t) \\
& \leq \Upsilon_{0}(t) \leq \frac{\max \left\{3,2+\bar{D} r_{1}+\bar{D} r_{3}, r_{2}+r_{4}, r_{5}\right\}}{\min \left\{\underline{\lambda}\left(P_{0}\right), b_{0} \underline{\lambda}\left(P_{1}\right), b_{1}\right\}} V(t) .
\end{aligned}
$$


The existence of two positive constants $\rho$ and $R$ such that (10) holds directly follows. This concludes the proof of the Lemma.

\section{Control Design}

In this section, we now establish a sufficient condition guaranteeing (9) when the delay varies according to the integral relation (2). This new condition bears on the initial conditions (accordingly to the choice of the control gain).

Theorem 1: Consider the closed-loop system

$$
\begin{aligned}
& \dot{X}(t)=A X(t)+B \varphi(t-D(t)) \\
& Y(t)=C X(t) \\
& \dot{\hat{X}}(t)=A \hat{X}(t)+B \varphi(t-D(t))-L(Y(t)-C \hat{X}(t)) \\
& \int_{t-D(t)}^{t} \varphi(s) d s=1 \quad \text { with } \varphi(t)=\operatorname{Sat}_{[\underline{u},+\infty)}(u(t)) \\
& u(t)=u^{r}+K\left[e^{A D(t)} \hat{X}(t)+\int_{t-D(t)}^{t} e^{A(t-s)} B \varphi(s) d s-X^{r}\right]
\end{aligned}
$$

where $A$ and $B$ are defined in (6), $K$ is such that $A+B K$ is Hurwitz, $L$ is such that $A+L C$ is Hurwitz and with distinct eigenvalues, $X^{r}$ is the state equilibrium corresponding to the original equilibrium $x^{r}$ of plant (1) and $u^{r}$ is the corresponding (constant) control reference. Consider the functionals

$$
\begin{aligned}
\Theta(t)= & |X(t)-\hat{X}(t)| \\
& +\max _{s \in[t-\bar{D}, t]}\left|\left[\begin{array}{ll}
u(s)-u^{r} \quad \dot{u}(s) \ldots u^{(n-1)}(s)
\end{array}\right]^{T}\right| \\
\Upsilon(t)= & \left|X(t)-X^{r}\right|^{2}+|X(t)-\hat{X}(t)|^{2}+\int_{t-D(t)}^{t}\left(u(s)-u^{r}\right)^{2} d s \\
& +D(t)^{2} \int_{t-D(t)}^{t} \dot{u}(s)^{2} d s .
\end{aligned}
$$

Then, provided that $u_{0} \in \mathcal{C}^{n}([-\bar{D}, 0], \mathbb{R})$, there exists $\theta$ : $\mathbb{R}^{n} \mapsto \mathbb{R}_{+}^{*}$ such that if $\Theta(0)<\theta(K)$ condition (9) is fulfilled and the plant exponentially converges in the sense that there exist $R, \rho>0$ such that

$$
\forall t \geq 0, \Upsilon(t) \leq R \Upsilon(0) e^{-\rho t} .
$$

This result has a relatively direct interpretation: the previously presented Lemma 1 requires the delay to vary sufficiently slowly, while, on the other hand, here the delay variations implicitly depend on the control input through the integral (2), with variations which aggressiveness is scaled by the gain $K$. Then, restricting the input variations by choosing the initial conditions close enough to the desired equilibrium and in compliance with the feedback gain magnitude seems like a natural requirement.

The behavior of the function $\theta$ with respect to its argument $K$ could be investigated in future works but is not pursued here. A constructive expression of this function is given through (27), (84) and (90). From this expression, the study would involve to analyze the solution of the Lyapunov equation and the behavior of its eigenvalues with respect to $K$ variations. One could reasonably assume that $\theta(K) \rightarrow 0$ while $K \rightarrow-\infty$ (as $r(K)$ is expected in this case to tend to $\infty$ ). Therefore, for given initial conditions, the magnitude of the feedback gain should be chosen accordingly. For relatively large initial conditions, this would imply to use a small feedback gain, as illustrated below in Section IV. Therefore, this result can be interpreted as a small-gain condition.

Also, one can note that the requirement $u_{0} \in \mathcal{C}^{n}([-\underline{D}, 0], \mathbb{R})$ implies that the control is continuous at $t=0$, that is $u_{[-\underline{D}, 0)}$ is consistent with the value $u(0)$ imposed by the prediction-based control law (41). Such a condition can seem demanding from an application point of view, but, as emphasized in the illustration section, is not necessary in practice. Future developments will focus on the extension of this result to handle discontinuities at $t=0$.

Finally, we wish to compare our result to robustness approaches proposed, e.g, in [6], [20]. At first glance, it could seem that Lemma 1 in which the proposed result is built on is somehow restrictive in the sense that it requires the delay rate to be sufficiently small. In the two provided references, a nominal value of the delay is used and stabilization is guaranteed provided less demanding assumption on the delay disturbances. However, we would like to emphasize two points. First, for the considered integral relation (2), recasting the input-dependency as a delay perturbation does not seem like a natural move. Second, even if one decides to pick the nominal value $D^{r}=$ $1 / u^{r}$, one cannot a priori check that the delay disturbance $\delta(t)=D(t)-D^{r}$ will satisfy the conditions of [6]. The same holds for the requirement on the size of the delay perturbation established in [20] which may not be fulfilled. Guaranteeing that one of these conditions holds for $D(t)-D^{r}$ requires a deeper analysis such as the one proposed in the sequel. Finally, by using the exact current delay value $D(t)$ in the prediction in lieu of a nominal one ( $D^{r}$ for instance), we aim at improving delay compensation and therefore transient performance. We illustrate this point in simulations in Section IV.

We now detail the proof of Theorem 1.

\section{A. Relating the Delay Variations to Input Variations}

Taking a time-derivative of (40) and defining the error variable

$$
\varepsilon \triangleq \varphi-u^{r}
$$

one gets, using $\varphi \geq \underline{u}$

$$
|\dot{D}(t)|=\left|1-\frac{\varepsilon(t)+u^{r}}{\varepsilon(t-D(t))+u^{r}}\right| \leq \frac{2 \max \left|\varepsilon_{t}\right|}{\underline{u}} .
$$


As a result, condition (9) is satisfied if

$$
\forall t \geq 0, \quad \max \left|\varepsilon_{t}\right|<\frac{\underline{u} \Delta^{*}(K)}{2} .
$$

This is the condition we now focus on. It yields to the analysis of the dynamics of the variable $\varepsilon$. Before properly starting this analysis, we recall and extend some well-known stability results for DDE.

\section{B. Preliminary Results: Extension of the Halanay Inequality for Delay Differential Equations of Order $n \geq 1$}

We first recall the following result [15], [18] ${ }^{7}$ which was generalized in recent works [23], [34].

Lemma 2 (Halanay Inequality): Consider a positive continuous real-valued function $x$ such that

$$
\dot{x}(t) \leq-a x(t)+b \max x_{t}, \quad t \geq 0
$$

with $a \geq b \geq 0$. Then, there exists $\gamma \geq 0$ such that

$$
\forall t \geq 0, \quad x(t) \leq \max x_{0} e^{-\gamma t} .
$$

A straightforward extension of this lemma is stated in the corollary below.

Corollary 1: Consider a positive continuous function such that

$$
\left\{\begin{array}{l}
\dot{x}(t) \leq-a x(t)+b h(t)+c e^{-\gamma t}, \quad t \geq 0 \\
x_{0}=\psi \in \mathcal{C}^{0}\left([-\bar{D}, 0], \mathbb{R}_{+}\right)
\end{array}\right.
$$

where $a \geq b \geq 0, \gamma>0$ and $h$ is a continuous functional satisfying, for some $\omega>0$, the sup-norm relation

$$
|h(t)| \leq \max \left|x_{t}\right|, \quad \text { for } \max \left|x_{t}\right|<\omega .
$$

Then, if the initial condition is such that $\max \psi<\omega$, there exists $c_{\omega}^{*}>0$ (depending on $\omega$ ) such that, if $|c|<c_{\omega}^{\star}$, then there exists $\tilde{\gamma} \in[0, \gamma)(\tilde{\gamma}=0$ if $a=b$ and $\tilde{\gamma}>0$ otherwise) such that

$$
\forall t \geq 0, \quad x(t) \leq \max \left\{\max x_{0}, \mu^{\star}\left(|c|, \gamma, \gamma_{0}\right)\right\} e^{-\tilde{\gamma} t}
$$

in which $\mu^{\star}$ is defined in terms of $\gamma_{0}$, the unique non-negative solution of the equation $\gamma_{0}-a+b e^{\gamma_{0} \underline{D}}=0$, as

$$
\begin{aligned}
& \mu^{\star}\left(|c|, \gamma, \gamma_{0}\right) \\
& \quad=|c| \begin{cases}0 & \text { if } \gamma_{0}=0 \\
\max \left\{\frac{1}{a-b e^{\gamma \bar{D}}-\gamma}, \frac{1-\frac{\gamma}{\gamma_{0}}}{a-b e^{\gamma \bar{D}}-\gamma}\right\} \geq 0 & \text { if } \gamma \neq \gamma_{0} \\
\frac{1}{\gamma\left(1+b \bar{D} e^{\gamma \bar{D}}\right)} & \text { otherwise. }\end{cases}
\end{aligned}
$$

In the particular case $\omega=+\infty$, one can notice from the elements of proof detailed below that $c_{\omega}^{\star}=+\infty$, i.e., this result holds for any value of $c \in \mathbb{R}$, thanks to the global inequality (51).

\footnotetext{
${ }^{7}$ More precisely, in [15], this result is stated for $a>b>0$.
}

Proof: Consider $x$ a non-trivial positive and continuous solution ${ }^{8}$ of (50), with $\max \psi<\omega$. On a certain neighborhood (at least) of the origin $t=0$, from (51) and by continuity, it satisfies the inequality

$$
\dot{x}(t) \leq-a x(t)+b \max x_{t}+|c| e^{-\gamma t} .
$$

Following the spirit of the original proof in [15], we first consider a positive solution of the corresponding differential equation

$$
\dot{y}(t)=-a y(t)+b \max y_{t}+|c| e^{-\gamma t}, \quad t \geq 0
$$

with a given initial condition such that $y(0)=\mu>0$, where the constant $\mu$ will be chosen adequately below. Defining $\gamma_{0}$ as the unique non-negative solution of the equation $\gamma_{0}-a+b e^{\gamma_{0}} \underline{D}=$ 0 , some computations show that a suitable function $y$ is

$$
\begin{aligned}
\forall t & \in \mathbb{R}, y(t) \\
& = \begin{cases}\frac{|c|}{a-b e^{\gamma \bar{D}}-\gamma} e^{-\gamma t}+\left(\mu-\frac{|c|}{a-b e^{\gamma \bar{D}}-\gamma}\right) e^{-\gamma_{0} t} & \text { if } \gamma \neq \gamma_{0} \\
\left(\frac{|c|}{1+b \bar{D} e^{\gamma \bar{D}}} t+\mu\right) e^{-\gamma t} & \text { otherwise }\end{cases}
\end{aligned}
$$

which is positive and decreasing by choosing (if $\gamma_{0}>0$ ) $\mu>$ $\mu^{\star}\left(|c|, \gamma, \gamma_{0}\right)$ where $\mu^{\star}$ is defined in (53). ${ }^{9}$ One can also show that there exists $\tilde{\gamma} \in\left[0, \min \left\{\gamma, \gamma_{0}\right\}\right)$ such that

$$
\forall t \geq 0, \quad y(t) \leq \mu e^{-\tilde{\gamma} t} .
$$

Indeed, $(i)$ when $\gamma_{0} \neq \gamma$, this directly follows from the expression (56) and (ii) when $\gamma_{0}=\gamma, \tilde{\gamma} \geq 0$ exists as $\mu>\mu^{\star}(|c|, \gamma)$. In details, if $\gamma_{0}>0$ (i.e., $a>b$ ), there exists $\tilde{\gamma}>0$ and $\tilde{\gamma}=0$ otherwise.

Now, define the difference $z=y-x$ which is a continuous function. We aim at choosing the initial condition of $y$ such that $z(t)>0$ for $t \in[-\underline{D}, 0]$, i.e., $\mu>\max x_{0}$. Further, to take advantage of (51), we would like to guarantee that $\max y(t)<\omega$ for all $t \geq 0$. Gathering all these conditions, this finally implies to choose $\mu \in(0, \omega) \cap\left(\max \left\{\max x_{0}, \mu^{\star}(c)\right\}, \infty\right)$. Provided $\mu^{\star}(|c|)<\omega$, this set is non-empty as $\max x_{0}<\omega$. Let us now discuss this assumption. For given parameters $a, b$ and $\gamma, \mu^{\star}$ is a linear function of $|c|$. Therefore, there exists $c_{\omega}^{\star}>0$ such that, for $|c|<c_{\omega}^{*}, \mu^{\star}(|c|)<\omega$ and the choice of $\mu$ is valid.

We are now interested in the (potential) sign change of $z$. As the function $z$ is continuous, we consider

$$
t_{1}=\inf \{t>0 \mid z(t)=0\} \in \mathbb{R} .
$$

Assume that $t_{1}$ is well-defined. From its definition, $z(t)>0$ for $t \in\left[0, t_{1}\right)$ and, from the analytical expression of $y$ and as both

${ }^{8}$ The existence of such a continuous function is obtained by considering the equality corresponding to (50) for which continuous (and even more) solutions exist assuming the initial condition $\psi$ smooth enough [2]. Such a solution is then also a solution of the inequality (50).

${ }^{9} \mu^{\star}$ is always positive for $\gamma_{0}>0$. Indeed, if $\gamma \neq \gamma_{0}$, the second coefficient involved in its definition through the max function is strictly positive. Specifically, for $\gamma<\gamma_{0}, a-b e^{\gamma \bar{D}}-\gamma>0$ and $1-\gamma / \gamma_{0}>0$ and, for $\gamma>\gamma_{0}$, $a-b e^{\gamma \bar{D}}-\gamma<0$ and $1-\gamma / \gamma_{0}<0$. 
$x$ and $y$ are continuous, $x(t)<y(t) \leq \mu<\omega$ for $t \in\left[0, t_{1}\right)$. Hence, the following inequality holds:

$$
\forall t \in\left[0, t_{1}\right), \quad \dot{z}(t)+a z(t) \geq b\left(\max y_{t}-\max x_{t}\right) .
$$

Then, $\dot{z}\left(t_{1}\right) \geq \max y_{t_{1}}-\max x_{t_{1}}>0$, by definition of $t_{1}$. Yet, one has

$$
\dot{z}\left(t_{1}\right)=\lim _{t \rightarrow t_{1}^{-}} \frac{z(t)-z\left(t_{1}\right)}{t-t_{1}}=\lim _{t \rightarrow t_{1}^{-}} \frac{z(t)}{t-t_{1}} \leq 0
$$

as $z(t) \geq 0$ on $\left[0, t_{1}\right]$. This contradiction allows us to conclude that such an instant $t_{1}$ does not exist and that, $\forall t \geq 0 z(t)>0$. Finally, using (57)

$$
\forall t \geq t_{0} \quad x(t) \leq \max \left\{\max x_{t_{0}}, \mu^{\star}\left(|c|, \gamma, \gamma_{0}\right)\right\} e^{-\tilde{\gamma} t}
$$

which is the desired result.

Lemma 3 (Stability of $a n^{\text {th }}$ Order DDE): Let $x$ be a solution of the $n^{\text {th }}$ order DDE

$$
\left\{\begin{array}{l}
x^{(n)}+\alpha_{n-1} x^{(n-1)}+\ldots+\alpha_{0} x= \\
\beta_{1} \ell_{1}\left(t, x_{t}, \ldots x_{t}^{(n-1)}\right)+\beta_{2} \ell_{2}(t), \quad \text { for } t \geq 0 \\
{\left[x \ldots x^{(n-1)}\right]_{0}^{T}=\psi \in \mathcal{C}^{0}\left([-\bar{D}, 0], \mathbb{R}^{n}\right) \text { with } \max |\psi|<\omega}
\end{array}\right.
$$

where the left-hand side of the differential equation defines a polynomial function the roots of which have strictly negative real part, $\omega>0, \beta_{1} \geq 0, \beta_{2}$ is a scalar constant and $\ell_{1}$ and $\ell_{2}$ are continuous functionals. There exist $\beta_{\max }^{1}>0, \beta_{\max }^{2}>0$ and $r_{\star}>0\left(\beta_{\max }^{1}=\alpha_{0}, \beta_{\max }^{2}=\infty\right.$ and $r_{\star}=1$ if $\left.n=1\right)$ such that, if

1) $0 \leq \beta_{1}<\beta_{\max }^{1}$ and $\left|\beta_{2}\right|<\beta_{\max }^{2}$;

2) there exists $\gamma>0$ such that $\left|\ell_{2}(t)\right| \leq e^{-\gamma t}$ for $t \geq 0$;

3) $\ell_{1}$ satisfies the following sup-norm relation, with $X \triangleq$ $\left[x \dot{x} \ldots x^{(n-1)}\right]^{T}$

$\forall t \geq t_{0}, \quad\left|\ell_{1}\left(t, x_{t}, \ldots, x_{t}^{(n-1)}\right)\right| \leq \max \left|X_{t}\right|$

for $t \geq 0, \max \left|X_{t}\right|<r \omega$ with $r \geq r_{\star}$

then, there exists $\tilde{\gamma} \in(0, \gamma)$ and $R>0$ such that

$$
\forall t \geq 0, \quad|X(t)| \leq \max \left\{r_{\star} \max \left|X_{0}\right|, R \beta_{2}\right\} e^{-\tilde{\gamma} t} .
$$

Proof: The idea is to use the scalar result of Corollary 1 Define the scalar-valued function $m(t) \triangleq X(t)^{T} P X(t)$ where $P$ is, as defined in the statement of the Lemma, the symmetric positive definite matrix solution of the Lyapunov equation $A_{0}^{T} P+P A_{0}=-Q$, for some given symmetric positive definite matrix $Q$, and $A_{0}$ is the companion matrix

$$
A_{0}=\left(\begin{array}{cccc}
0 & 1 & & \\
\vdots & & \ddots & \\
0 & & & 1 \\
-\alpha_{0} & -\alpha_{1} & \ldots & -\alpha_{n-1}
\end{array}\right) .
$$

Define $a \triangleq(\underline{\lambda}(Q) / 2 \bar{\lambda}(P)), \quad b \triangleq 2 \beta_{1}(\bar{\lambda}(P) / \underline{\lambda}(P))$ and $c=$ $\left(2 \beta_{2}^{2} \bar{\lambda}(P)^{2} / \underline{\lambda}(Q)\right)$. Taking a time-derivative of $m$ and, classically, applying Young's inequality, one can obtain

$$
\dot{m}(t) \leq-a m(t)+b h(t)+c e^{-2 \gamma t}
$$

with

$$
\begin{aligned}
h(t) & =\frac{\underline{\lambda}(P)}{\beta_{1} \bar{\lambda}(P)} X(t)^{T} P\left(\begin{array}{c}
0 \\
\vdots \\
0 \\
\beta_{1} \ell_{1}(.)
\end{array}\right) \\
& \leq \sqrt{\underline{\lambda}(P)} \sqrt{m(t)}\left|\ell_{1}\left(t, x_{t}, \ldots, x_{t}^{(n-1)}\right)\right|
\end{aligned}
$$

which satisfies, for $\max \left|X_{t}\right|<r \omega$ (and therefore, in particular, for $\left.\max m_{t}<r^{2} \omega^{2} \underline{\lambda}(P)\right)$

$$
|h(t)| \leq \sqrt{m(t)} \max \sqrt{m_{t}} \leq \max m_{t} .
$$

Define the condition

$$
\max m_{0}<r^{2} \omega^{2} \underline{\lambda}(P) .
$$

If (69) holds, then one concludes, applying Corollary 1 , that there exists $c_{\omega}^{\star}>0$ such that, if $|c|<c_{\omega}^{\star}$ and $a>b$, then there exists $\tilde{\gamma} \in(0, \gamma)$ such that

$$
\forall t \geq 0, \quad m(t) \leq \max \left\{\max \left|m_{t_{0}}\right|, \mu^{*}\right\} e^{-2 \tilde{\gamma} t}
$$

in which $\mu^{*}$ is defined in (53). Yet, a sufficient condition for $\max m_{0}<r^{2} \omega^{2} \lambda(P)$ to hold is that $\max \left|X_{0}\right|^{2}<$ $r^{2} \omega^{2}(\underline{\lambda}(P) / \bar{\lambda}(P))$. By assumption, $\max \left|X_{0}\right|<\omega$. Hence, to guarantee that (69) holds, one simply has to pick $r \geq r_{\star} \triangleq$ $\sqrt{\bar{\lambda}(P) / \underline{\lambda}(P)}$. Finally, the condition $a>b$ can be reformulated as $\beta_{1}<\left(\underline{\lambda}(P) \underline{\lambda}(Q) / 4 \bar{\lambda}(P)^{2}\right) \triangleq \beta_{\text {max }}^{1}$, the condition $|c|<c_{\omega}^{\star}$ as $\left.\left|\beta_{2}\right|<\sqrt{c_{\omega}^{*} \underline{\lambda}(Q) / 2 \bar{\lambda}(P)^{2}}\right) \triangleq \beta_{\text {max }}^{2}$ and one obtains

$\forall t \geq t_{0},|X(t)| \leq \max \left\{\sqrt{\frac{\bar{\lambda}(P)}{\underline{\lambda}(P)}} \max \left|X_{0}\right|, \sqrt{\frac{\mu^{*}}{\underline{\lambda}(P)}}\right\} e^{-\tilde{\gamma} t}$

which concludes the proof.

\section{Proof of Theorem 1-Application of Halanay-Like Inequality to Dynamical Equation of the Input Error Variable $\varepsilon$}

To obtain a sufficient condition guaranteeing (47), we first focus on the DDE governing $\varepsilon$, which is given in the following lemma.

Lemma 4: Consider $t_{0} \in \mathbb{R}$ and assume that the function $\varphi$ is unsaturated for $t \leq t_{0}$ (or equivalently that $u(t) \geq \underline{u}, t \leq t_{0}$ ). Provided that $u_{0} \in \mathcal{C}^{n}([-\bar{D}, 0], \mathbb{R})$, the error variable $\varepsilon=u-$ $u^{r}$ with $u$ defined in (41) is $n$ times continuously differentiable for $t \geq t_{0}$ and satisfies the following differential equation for $t \geq t_{0}$

$$
\begin{aligned}
\varepsilon^{(n)} & +\left(a_{n-1}+b_{0} k_{n-1}\right) \varepsilon^{(n-1)}+\ldots+\left(a_{0}+b_{0} k_{0}\right) \varepsilon \\
= & \pi_{1}\left(\dot{D}, \ldots, D^{(n)}, \varepsilon_{t}, \ldots, \varepsilon_{t}^{(n-1)}, \tilde{X}, \frac{1}{1+\dot{D}}\right) \\
& +K \pi_{2}\left(\dot{D}, \ldots, D^{(n)}\right) \tilde{X}(t)
\end{aligned}
$$


where the constants $k_{i}(i \in\{1, \ldots, n-1\})$ are such that $\left[-k_{0} \ldots-k_{n-1}\right] \triangleq K, \pi_{1}$ and $\pi_{2}$ are polynomial functions and $\pi_{1}$ is at least quadratic in the variables $\dot{D}, \ldots, D^{(n)}$, $\varepsilon_{t}, \ldots, \varepsilon_{t}^{(n-1)}, \tilde{X}$.

Proof: For sake of clarity, the proof of this theorem is given in Appendix.

Now that we are equipped with this last lemma, it is possible to use Lemma 3 to guarantee that the stability condition (47) holds.

Lemma 5: Consider the functional $\Theta$ defined in (42). Provided that $u_{0} \in \mathcal{C}^{n}([-\bar{D}, 0], \mathbb{R})$, there exists $\theta: \mathbb{R}^{n} \mapsto \mathbb{R}_{+}^{*}$ such that, if $\Theta(0)<\theta(K)$, then

$$
\forall t \geq 0, \quad|\varepsilon(t)| \leq \min \left\{\frac{\underline{u} \Delta^{\star}(K)}{2}, u^{r}-\underline{u}\right\}
$$

which implies that condition (47) is fulfilled.

Proof: Assume for a moment that the function $\varphi$ is not saturated for $t \leq 0$. Then, dynamics (72) holds and is compliant with the assumptions of Lemma 3.

In details, first, the left-hand side of (72) is asymptotically stable, as it represents the last line of the Hurwitz companion matrix $A+B K$. Second, by observing that

$$
\dot{D}=\frac{\varepsilon(t-D)-\varepsilon(t)}{\varepsilon(t-D)+u^{r}}
$$

one can obtain by induction that, for $m \geq 1, D^{(m)}$ is a polynomial function in $\varepsilon_{t}, \ldots, \varepsilon_{t}^{(m-1)}, 1 / \varepsilon(t-D)+u^{r}$ without any term of order 0 or 1 . Therefore, $\pi_{1}$ is directly a polynomial function of the variables $\varepsilon_{t}, \ldots, \varepsilon_{t}^{(n-1)}, \tilde{X},(1 / 1+$ $\dot{D}),\left(1 / \varepsilon(t-D)+u^{r}\right)$, which is at least quadratic in the variables $\varepsilon_{t}, \ldots, \varepsilon_{t}^{(n-1)}, \tilde{X}$ and $\pi_{2}$ is a polynomial function in $\epsilon_{t}, \ldots, \epsilon_{t}^{(n-1)},\left(1 / \varepsilon(t-D)+u^{r}\right)$.

Now, we distinguish some of the terms in $\pi_{1}$ and $\pi_{2}$. First, consider $c_{2,0}$ the constant term of $\pi_{2}$ and $c_{2,1}$ the term of first order in $\pi_{2}$ involving $1 / \varepsilon(t-D)+u^{r}$. Further, consider $c_{1,2}$ the terms in $\pi_{1}$ involving $\tilde{X}$ at power 1 and only one of the variables $\epsilon_{t}, \ldots, \epsilon_{t}^{(n-1)}$ and at power 1 . This term can be written as follows:

$$
c_{1,2}=\pi_{3}\left(\varepsilon_{t}, \ldots, \varepsilon_{t}^{n-1}, \frac{1}{1+\dot{D}}, \frac{1}{\varepsilon(t-D)+u^{r}}\right) \tilde{X}
$$

in which $\pi_{3}$ is a (vectorial) polynomial function the terms of which involve exactly one of the variables $\varepsilon_{t}, \ldots, \varepsilon_{t}^{n-1}$. Now, applying Young's inequality, one can obtain

$\left|c_{1,2}\right| \leq \frac{1}{2}\left|\pi_{3}\left(\varepsilon_{t}, \ldots, \varepsilon_{t}^{n-1}, \frac{1}{1+\dot{D}}, \frac{1}{\varepsilon(t-D)+u^{r}}\right)\right|^{2}+\frac{|\tilde{X}(t)|^{2}}{2}$

in which $\left|\pi_{3}\right|^{2}$ is now a polynomial function at least quadratic in $\varepsilon_{t}, \ldots, \varepsilon_{t}^{n-1}$.
We are now ready to define the functionals $\ell_{1}$ and $\ell_{2}$ considered in Lemma 3. Provided that the initial state estimate $\tilde{X}(0)$ is not equal to zero, define

$$
\begin{aligned}
& \beta_{2}=|K|\left(\left|c_{2,0}\right|+\left|c_{2,1}\left(\frac{1}{\underline{u}}\right)\right|\right)|\tilde{X}(0)|+\frac{|\tilde{X}(0)|^{2}}{2} \\
& \ell_{2}(t)=\frac{1}{\beta_{2}}\left[K\left(c_{2,0}+c_{2,1}\left(\frac{1}{\varepsilon(t-D)+u^{r}}\right)\right) \tilde{X}(t)+\frac{|\tilde{X}(t)|^{2}}{2}\right]_{(78)}^{(77)} \\
& \beta_{1} \ell_{1}(.)=\frac{1}{2}\left|\pi_{3}\left(\varepsilon_{t}, \ldots, \varepsilon_{t}^{(n-1)}, \frac{1}{1+\dot{D}}, \frac{1}{\varepsilon(t-D)+u^{r}}\right)\right|^{2} \\
& +\left(\pi_{1}-c_{1,2}\right)\left(\varepsilon_{t}, \ldots, \varepsilon_{t}^{(n-1)}\right) \\
& +K\left[\pi_{2}\left(\varepsilon_{t}, \ldots, \varepsilon_{t}^{(n-1)}, \tilde{X}, \frac{1}{1+\dot{D}}, \frac{1}{\varepsilon(t-D)+u^{r}}\right)-c_{2,0}\right. \\
& \left.\quad-c_{2,1}\left(\frac{1}{\varepsilon(t-D)+u^{r}}\right)\right] \tilde{X}(t) .
\end{aligned}
$$

Alternatively, if $|\tilde{X}(0)|=0$, one can define $\beta_{2}=0$ and $\ell_{2}=0$.

We now prove that conditions 1 ) to 3 ) of Lemma 3 hold. Indeed, from the error equation of $\tilde{X}$ given in (95), one can actually obtain that

$$
\forall t \geq 0, \quad|\tilde{X}(t)| \leq|\tilde{X}(0)| e^{\underline{\lambda}(A+L C) t}
$$

if $L$ is chosen such that the eigenvalues of $A+L C$ are distinct and in which $\underline{\lambda}(A+L C)<0$. From the previous definitions, using now the fact that $\left|\left(1 / \epsilon(t-D)+u^{r}\right)\right| \leq(1 / \underline{u})$, one can get that $\left|\ell_{2}(t)\right| \leq e^{\underline{\lambda}(A+L C) t}$ for $t \geq 0$. Further, one can observe

$$
\left|\beta_{1} \ell_{1}\right| \leq \frac{1}{2}\left|\pi_{3}\right|^{2}+\left|\pi_{1}-c_{1,2}\right|+|K|\left|\pi_{2}-c_{2,0}-c_{2,1}\right| \tilde{X}(0) \mid
$$

in which $\left|\pi_{3}\right|^{2},\left|\pi_{1}-c_{1,2}\right|$ and $\left|\pi_{2}-c_{2,0}-c_{2,1}\right|$ are polynomial functions at least quadratic in $\varepsilon_{t}, \ldots, \varepsilon_{t}^{(n-1)}$. Now, from the previous observation on $\left|\left(1 / \epsilon(t-D)+u^{r}\right)\right|$, and observing that

$$
\frac{1}{1+\dot{D}}=\frac{\varepsilon(t-D)+u^{r}}{2 \varepsilon(t-D)-\varepsilon(t)+u^{r}}
$$

it is possible to properly define a neighborhood of the origin $\Omega \triangleq(-\omega(K), \omega(K))$ in which $\beta_{1} \ell_{1}$ satisfies the sup-norm

$$
\left|\beta_{1} \ell_{1}(\cdot)\right| \leq \beta_{\max }^{1} \max \left|E_{t}\right|
$$

in which $E \triangleq\left[\epsilon \dot{\epsilon} \ldots \epsilon^{(n-1)}\right]^{T}$. Note that this neighborhood depends both on the functional $\ell_{1}$ and on the constant $\beta_{\max }^{1}$ and therefore on the feedback gain $K$. Finally, one obtains that $\left|\beta_{2}\right|<\beta_{\max }^{2}$ if

$$
\begin{aligned}
|\tilde{X}(0)| & <\min \left\{1, \kappa(K) \beta_{\max }^{2}\right\} \triangleq \rho^{\star}(K, \omega(K)) \\
\kappa(K) & \triangleq \frac{1}{|K|\left(\left|c_{2,0}\right|+\left|c_{2,1}\left(\frac{1}{\underline{u}}\right)\right|\right)+\frac{1}{2}}
\end{aligned}
$$


in which $\beta_{\max }^{2}$, and therefore $\rho^{*}$, depend on $\omega(K)$. Therefore, for $\max E_{0}([-\underline{D}, 0])<\omega(K)$ and $|\tilde{X}(0)|<\rho^{\star}(K, \omega(K))$, Lemma 3 guarantees the existence of $r(K)>0, R(K)>0$ and $\tilde{\gamma}>0$ such that

$$
\forall t \geq 0, \quad|E(t)| \leq \max \left\{r \max \left|E_{0}\right|, R \beta_{2}\right\} e^{-\tilde{\gamma} t}
$$

as long as the actuator $\varphi$ is not saturated and because (72) applies. Consequently, if $\tilde{X}(0)$ satisfies the more restrictive condition

$$
|\tilde{X}(0)|<\min \left\{\rho^{*}(K, \omega(K)), \kappa(K) \frac{r(K)}{R(K)} \max \left|E_{0}\right|\right\}
$$

then, one obtains

$$
\forall t \geq 0, \quad|E(t)| \leq r(K) \max \left|E_{0}\right| e^{-\tilde{\gamma} t}
$$

as long as the actuator $\varphi$ is not saturated. Yet, one can observe that a sufficient condition to guarantee that the actuator is not saturated is $|\varepsilon(t)| \leq u^{r}-\underline{u}, t \geq 0$. Therefore, by choosing

$$
\max \left|E_{0}\right| \leq \frac{1}{r(K)} \min \left\{\frac{\underline{u} \Delta^{*}(K)}{2}, u^{r}-\underline{u}, \omega(K)\right\}
$$

one can ensure together that this condition is fulfilled for any $t \geq 0$, that the initial condition lies in the neighborhood $\Omega$ and that $|E(t)| \leq\left(\underline{u} \Delta^{*}(K) / 2\right), t \geq 0$. In particular, the condition (47) is also fulfilled.

Finally, the two choices bearing on $\max \left|E_{0}\right|$ and $|\tilde{X}(0)|$ can be expressed in terms of $\Theta$ by defining

$$
\begin{aligned}
& \theta(K) \triangleq \frac{1}{r(K)} \min \left\{\frac{\underline{u} \Delta^{*}(K)}{2}, u^{r}-\underline{u}, \omega(K)\right\} \\
& +\min \left\{\rho^{*}(K, \omega(K)), \frac{\kappa(K)}{R(K)} \min \left\{\frac{\underline{u} \Delta^{*}(K)}{2}, u^{r}-\underline{u}, \omega(K)\right\}\right\} .
\end{aligned}
$$

This gives the conclusion.

The proof of Theorem 1 directly follows from Lemma 5.

\section{ILlustrative EXAMPLE}

In this section, for illustration purposes, we consider the following simple unstable second-order plant

$$
\left\{\begin{array}{l}
\ddot{x}-\dot{x}+x=\varphi(t-D(t)) \\
\int_{t-D(t)}^{t} \varphi(s) d s=1, \quad \varphi(t)=\operatorname{Sat}_{[0.01,+\infty)}(u(t)) .
\end{array}\right.
$$

Following the proposed methodology, we introduce the matrices

$$
A=\left(\begin{array}{cc}
0 & 1 \\
-1 & 1
\end{array}\right), \quad B=\left(\begin{array}{l}
0 \\
1
\end{array}\right)
$$

and implement the control law defined through (40), (41).

One can note that the chosen initial condition $u_{0}([-\underline{D}, 0), \mathbb{R})$ is not consistent with the value $u(0)$ imposed by the predictionbased control law, resulting into a discontinuity for $t=0$.

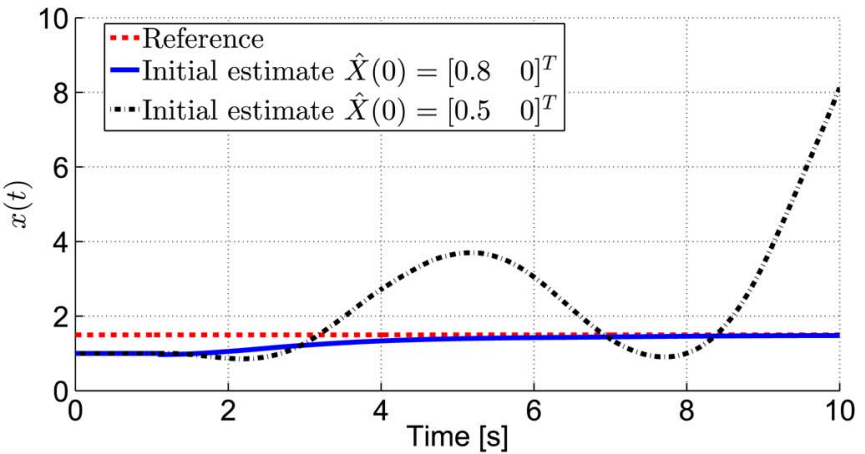

(a)

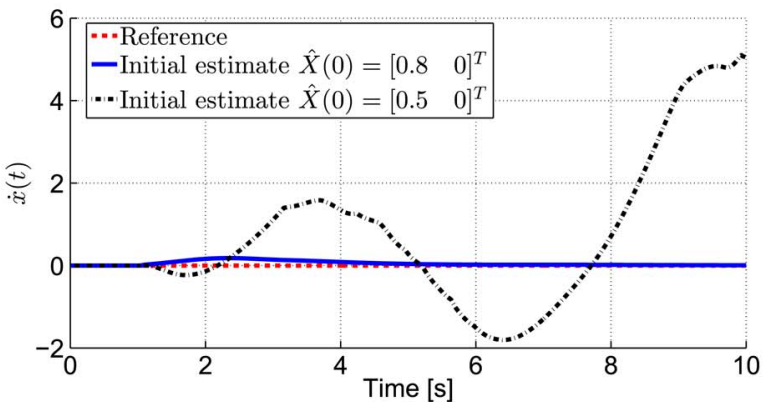

(b)

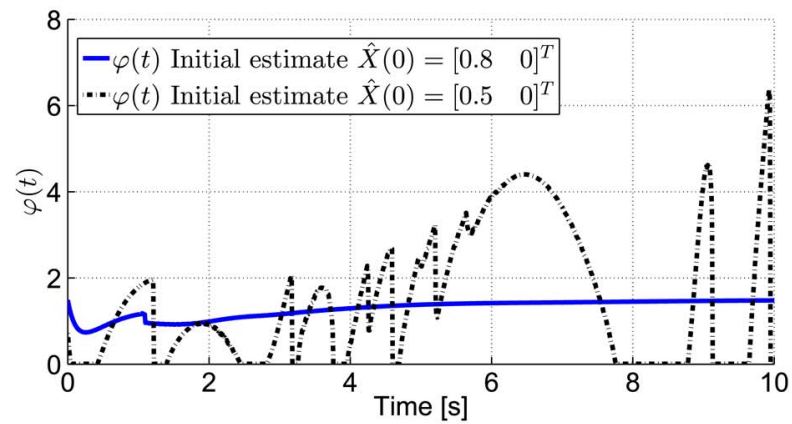

(c)

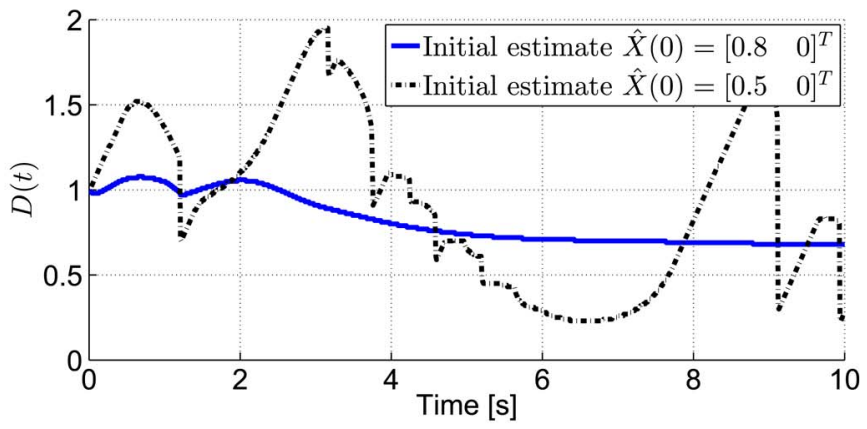

(d)

Fig. 3. Simulation results for the same feedback gain $\left(K=-\left[\begin{array}{ll}1 & 2\end{array}\right]\right)$ and two sets of initial conditions, respectively $\hat{X}(0)=\left[\begin{array}{ll}0.8 & 0\end{array}\right]^{T}$ and $\hat{X}(0)=\left[\begin{array}{ll}0.5 & 0\end{array}\right]^{T}$. (a) $x(t)$; (b) $\dot{x}(t)$; (c) Saturated control law; (d) Delay variations.

Hence, strictly speaking, the smoothness requirement of the Theorem 1 is not satisfied. However, this does not prevent the conclusion of Theorem 1 to hold, as detailed below. Further, it is worth noting that this condition could still be satisfied by employing an additive input filter.

Simulation results for two different sets of initial conditions, respectively $\hat{X}(0)=[0.80]^{T}$ and $\hat{X}(0)=[0.50]^{T}$ for $X(0)=$ $\left[\begin{array}{ll}1 & 0\end{array}\right]^{T}$, are pictured in Fig. 3. Initial control values are similar in 


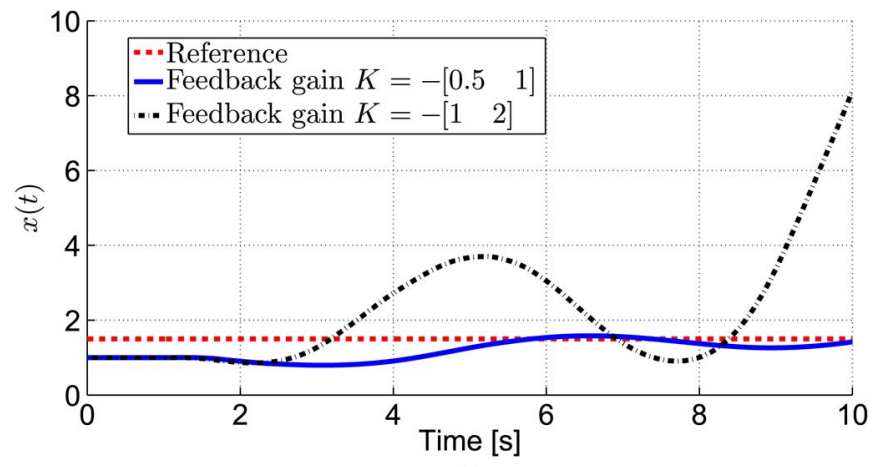

(a)

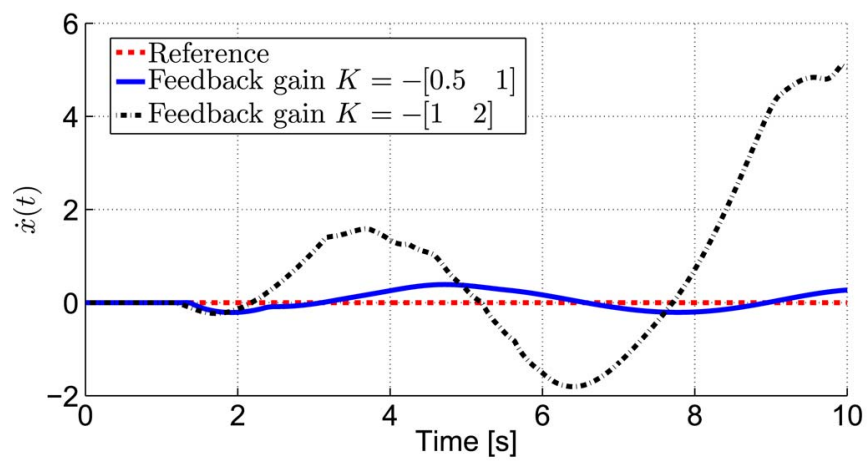

(b)

Fig. 4. Simulation results for the same initial conditions $\left(\hat{X}(0)=\left[\begin{array}{ll}0.5 & 0\end{array}\right]^{T}\right)$ and two values of the feedback gain respectively $K=-\left[\begin{array}{ll}0.5 & 1\end{array}\right]$ and $K=$ $-[12]$. (a) $x(t)$; (b) $\dot{x}(t)$

both cases $u(t)=1, t \in[-\underline{D}, 0]$, resulting into an initial delay $D(0)=1$ the effects of which can be noticed on the beginning of the state response. The control aims at stabilizing the plant at the target equilibrium $x^{r}=1.5$ and (stabilizing) feedback gain has been chosen as $K=-\left[\begin{array}{ll}1 & 2\end{array}\right]$. Observer gain is similar in the two cases $L=-\left[\begin{array}{ll}10 & 12\end{array}\right]$.

Obviously, one can observe that stabilization is achieved for the first set of initial conditions, while, for the second set, the initial estimation error is too important. These results emphasize the local nature of Theorem 1 and can be reasonably related to the delay variations pictured in Fig. $3 .{ }^{10}$ Indeed, the goal of Theorem 1 is to ensure that Condition (9) in Lemma 1, which restricts the delay variations, is satisfied. Here, for the second set of initial conditions, the initial state estimation error implies more important control fluctuations and therefore delay variations which are too important to be handled.

Nevertheless, for this set of initial conditions, it is still possible to change the magnitude of the feedback gain to increase the stabilization region defined through $\theta(K)$ in Theorem 1 so that it contains $\Theta(0)$. This is what is looked for in the simulations reported in Fig. 4 in which the initial conditions are similar to the second set previously considered $(\hat{X}(0)=$ $\left[\begin{array}{ll}0.5 & 0\end{array}\right]^{T}$ with the rest of the setting unchanged) and two different feedback gains are used, respectively $K=-\left[\begin{array}{ll}0.5 & 1\end{array}\right]$ (detuned controller) and $K=-\left[\begin{array}{ll}1 & 2]\end{array}\right]$ (both yielding a Hurwitz matrix $A+B K)$. Intuitively, pursuing the consideration pre-

\footnotetext{
${ }^{10}$ Alternatively, simulations were performed keeping the same initial state estimate but changing the state reference and lead to the same conclusions.
}

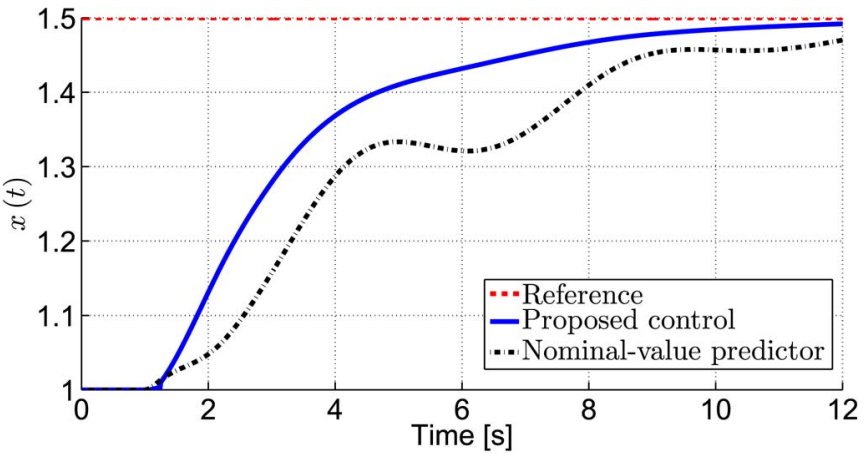

(a)

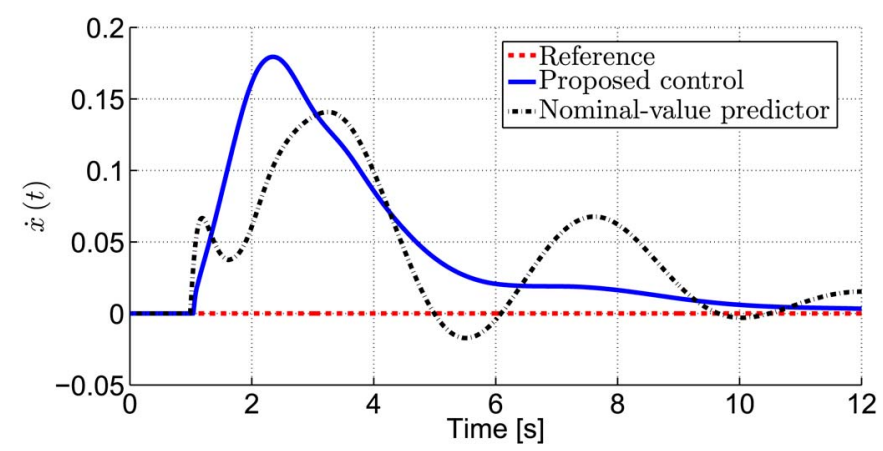

(b)

Fig. 5. Simulation results for the proposed prediction-based controller and one employing a nominal delay value $D^{r}=1 / u^{r}$ as prediction horizon. The initial conditions are the same $\left(\hat{X}(0)=\left[\begin{array}{ll}0.5 & 0\end{array}\right]^{T}\right)$ and the feedback gain is chosen as $K=-[12]$. (a) $x(t)$; (b) $\dot{x}(t)$.

sented earlier in Section III, the stabilization region could increase while decreasing $K$. Indeed, this is what is observed in simulation, as stabilization is obtained for $K=-\left[\begin{array}{ll}0.5 & 1\end{array}\right]$ while not for $K=-[12]$, as before. Therefore, for given initial condition, restricting the feedback gain magnitude may enable stabilization: the statement of Theorem 1 can be understood as a small-gain result.

Finally, to stress the interest of the proposed method compared to approaches recasting the delay input-variations as a disturbance, we also compare the performance of our controller with a prediction-based controller employing a nominal delay value $D^{r}=1 / u^{r}$ as prediction horizon. Simulation results are depicted in Fig. 5. One could reasonably expect that, by employing the current delay in lieu of its asymptotic value, the control law would consequently better compensate the input delay. Indeed, this is what could be observed in Fig. 5: both controllers achieve stabilization but the one proposed in this paper achieves smoother and better performance compared to the (relatively slight) oscillatory behavior of the second one. This gain is modest but one could reasonably hope to improve it by carefully tuning the controller gain.

\section{CONClusion}

In this paper, the problem of robust delay compensation of a linear system driven by a delayed input by means of output feedback has been addressed for a particular class of input-dependent delay defined through an integral relation. The proposed approach is based on a two-steps methodology, 
which, first, requires the delay variations to be bounded and, in turn, relates these variations to input fluctuations. The obtained sufficient conditions yield local asymptotic stability by putting limits on initial conditions depending on the feedback gain.

A natural extension of this work would be to study more complex integral-type delay defining equations, in which the integral kernel depends less directly on the input. A second interesting step would be to investigate the compliance of the considered prediction framework with other input-varying delay models, e.g., models of variable time lag appearing in combustion instability studies [17], [27].

\section{APPENDIX}

\section{PROOF OF LEMMA 4}

Before starting the actual proof, we establish preliminary results. In the following, we note $Z=\hat{X}-X^{r}$ the estimate state tracking error which satisfies, following (37) and (41)

$$
\left\{\begin{array}{l}
\dot{Z}(t)=A Z(t)+B \varepsilon(t-D(t))-L C \tilde{X}(t) \\
\varepsilon(t)=K\left[e^{A D(t)} Z(t)+\int_{t-D(t)}^{t} e^{A(t-s)} B \varepsilon(s) d s\right] \\
\dot{\tilde{X}}(t)=(A+L C) \tilde{X}(t)
\end{array}\right.
$$

when the actuator is not saturated over the whole time interval $[t-D(t), t]$.

\section{A. Preliminary Results}

Lemma 6: If the actuator is unsaturated over the interval $[t-$ $D(t), t]$ and if $u_{0} \in \mathcal{C}^{n}([-\bar{D}, 0], \mathbb{R})$, the control variable stated in (41) is $n$ times continuously differentiable and satisfies the following differential equations

$$
\text { for } \begin{aligned}
1 & \leq m \leq n, \varepsilon^{(m)}-\sum_{l=1}^{m} K A^{l-1} B \varepsilon^{(m-l)} \\
= & f_{Z}^{m}(t)+f_{\varepsilon}^{m}(t)+f_{\tilde{X}}^{m}(t)+(1+\dot{D})^{m} K e^{A D} A^{m} Z \\
& +K \int_{t-D}^{t} A^{m} e^{A(t-s)} B \varepsilon(s) d s
\end{aligned}
$$

with

$$
\begin{aligned}
& \left\{\begin{array}{l}
f_{Z}^{1}(t)=0 \text { and for } 2 \leq m \leq n \\
f_{Z}^{m}(t)=\frac{d\left[(1+\dot{D})^{m-1}\right]}{d t} K e^{A D} A^{m-1} Z+\frac{d}{d t}\left(f_{Z}^{m-1}(t)\right)
\end{array}\right. \\
& \left\{\begin{array}{l}
f_{\varepsilon}^{1}(t)=\dot{D} K e^{A D} B \varepsilon(t-D) \text { and for } 2 \leq m \leq n \\
f_{\varepsilon}^{m}(t)=\frac{d}{d t}\left(f_{\varepsilon}^{m-1}(t)\right) \\
\quad+\left[(1+\dot{D})^{m-1}-(1-\dot{D})\right] \varepsilon(t-D) K e^{A D} A^{m-1} B
\end{array}\right. \\
& \left\{\begin{array}{l}
f_{\tilde{X}}^{1}(t)=-K e^{A D} L C \tilde{X}(t) \text { and for } 2 \leq m \leq n \\
f_{\tilde{X}}^{m}(t)=\frac{d}{d t}\left(f_{\tilde{X}}^{m-1}(t)\right)-(1+\dot{D})^{m-1} K e^{A D} A^{m-1} L C \tilde{X}(t) .
\end{array}\right.
\end{aligned}
$$

Proof: The result is constructively obtained by induction and successive substitutions.

Initial step: one directly gets, taking a time-derivative of the control law (94) and using (93)

$$
\begin{aligned}
\dot{\varepsilon}(t) & =K B \varepsilon(t)+(1+\dot{D}) K e^{A D} A Z+\overbrace{\dot{D} K e^{A D} B \varepsilon(t-D)}^{=f_{\varepsilon}^{1}(t)} \\
+ & \overbrace{f_{Z}^{1}(t)}^{=0} \overbrace{-K e^{A D} L C \tilde{X}(t)}^{=f_{\tilde{X}}^{1}(t)}+K \int_{t-D}^{t} A e^{A(t-s)} B \varepsilon(s) d s
\end{aligned}
$$

which gives (96) for $m=1$. Further, as $u_{0} \in \mathcal{C}^{n}([-\bar{D}, 0], \mathbb{R})$, therefore $\varepsilon_{0} \in \mathcal{C}^{n}([-\bar{D}, 0], \mathbb{R})$. Further, $\varepsilon$ is continuous for $t \geq$ 0 according to the control law (94) and, following the expression of $\dot{D}$ given in (46), $\dot{D}$ is continuous. Therefore, one obtains that $\varepsilon$ is continuously differentiable for $t \geq 0$. This implies in particular that $\dot{D}$ is continuously differentiable according to (46) and, therefore, that $f_{\varepsilon}^{1}$ also is as $u_{0} \in \mathcal{C}^{1}([-\bar{D}, 0], \mathbb{R})$.

Induction: assume that the property is true for a given $m \geq$ 1 . We now show that it also holds for $m+1$. Taking a timederivative of (96) for some $m \geq 1$ yields

$$
\begin{aligned}
& \varepsilon^{(m+1)}-\sum_{l=1}^{m} K A^{l-1} B \varepsilon^{(m+1-l)}=\frac{d}{d t}\left(f_{\varepsilon}^{m}(t)\right) \\
& +\underbrace{\frac{d}{d t}\left(f_{Z}^{m}(t)\right)+\frac{d(1+\dot{D})^{m}}{d t} K e^{A D} A^{m} Z}_{=f_{Z}^{m+1}(t)}+K A^{m} B \varepsilon(t) \\
& +\dot{D}(1+\dot{D})^{m} K e^{A D} A^{m+1} Z+(1+\dot{D})^{m} K e^{A D} A^{m}[A Z \\
& +B \varepsilon(t-D)] \underbrace{-(1+\dot{D})^{m} K e^{A D} A^{m} L C \tilde{X}(t)+\frac{d}{d t}\left(f_{\tilde{X}}^{m}(t)\right)}_{=f_{\tilde{X}}^{m+1}(t)} \\
& -(1-\dot{D}) K e^{A D} A^{m} B \varepsilon(t-D)+K \int_{t-D}^{t} A^{m+1} e^{A(t-s)} B \varepsilon(s) d s .
\end{aligned}
$$

Rearranging terms, one obtains (96) for $m+1$. Similar considerations as those previously used yield that $\varepsilon$ is $m+1$ times continuously differentiable. The same holds for $D$ and one can show that $f_{\varepsilon}^{m+1}$ is continuously differentiable. This gives the conclusion.

Further, the sequences $\left(f_{\varepsilon}^{m}\right),\left(f_{Z}^{m}\right)$, and $\left(f_{\tilde{X}}^{m}\right)$ satisfy the following properties.

Lemma 7: For $2 \leq m \leq n$, the function $f_{\varepsilon}^{m}$ introduced in (98) in Lemma 6 is a polynomial function at least quadratic in $\varepsilon_{t}, \ldots, \varepsilon_{t}^{(m-1)}, \dot{D}, \ldots, D^{(m)}$.

Proof: The proof is straightforwardly obtained using the definition of $\left(f_{\varepsilon}^{m}\right)$ in Lemma 6 together with the fact that

$$
(1+\dot{D})^{m-1}-(1-\dot{D})=\sum_{l=1}^{m-1}\left(\begin{array}{l}
n \\
l
\end{array}\right) \dot{D}^{l}+\dot{D}
$$

which contains no degree 0 terms.

Lemma 8: For $1 \leq m \leq n$, the function $f_{\tilde{X}}^{m}$ introduced in (99) in Lemma 6 is in the following form:

$$
f_{\tilde{X}}^{m}=K \tilde{\pi}\left(\dot{D}, \ldots, D^{(m-1)}\right) \tilde{X}(t)
$$

with $\tilde{\pi}$ a polynomial function with values in $\mathcal{M}_{n}(\mathbb{R})$ 
Proof: This property directly follows from the definition of $\left(f_{\tilde{X}}^{m}\right)$ and (95).

Lemma 9: Assume that $1+\dot{D}>0$. For $2 \leq m \leq n$, the function $f_{Z}^{m}$ introduced in (97) in Lemma 6 is a polynomial function in $\varepsilon_{t}, \ldots, \varepsilon_{t}^{(m-1)}, \tilde{X}, \dot{D}, \ldots D^{(m)}$ and $1 / 1+\dot{D}$, at least quadratic in the variables $\varepsilon_{t}, \ldots, \varepsilon_{t}^{(m-1)}, \tilde{X}, \dot{D}, \ldots D^{(m)}$.

Proof: Again, we reason by induction.

Induction: we assume that the property is true for a given $m \geq 2$. Then, using (96) for $m$, one obtains

$$
\begin{aligned}
& f_{Z}^{m+1}(t)= \frac{d\left[(1+\dot{D})^{m}\right]}{d t} K e^{A D} A^{m} Z+\frac{d}{d t}\left(f_{Z}^{m}(t)\right) \\
&=\frac{m \ddot{D}}{1+\dot{D}}\left[\varepsilon^{(m)}-\sum_{l=1}^{m} K A^{l-1} \varepsilon^{(m-l)}-f_{Z}^{m}(t)-f_{\varepsilon}^{m}(t)\right. \\
&\left.\quad-f_{\tilde{X}}^{m}-K \int_{t-D}^{t} A^{m} e^{A(t-s)} B \varepsilon(s) d s\right]+\frac{d}{d t}\left(f_{Z}^{m}(t)\right) .
\end{aligned}
$$

Then, using the induction assumption jointly with the two previous lemmas, one can conclude that $f_{Z}^{m+1}$ is a polynomial function in $\tilde{X}, \varepsilon_{t}, \ldots, \varepsilon_{t}^{(m)}, \dot{D}, \ldots, D^{(m+1)},(1 / 1+\dot{D})$, at least quadratic in $\tilde{X}, \varepsilon_{t}, \ldots, \varepsilon_{t}^{(m)}, \dot{D}, \ldots, D^{(m+1)}$.

Initial step: the same arguments as above apply for $m=2$.

\section{B. Design of (72) Based on Lemma 6, 7, 9, and 8}

As the dynamics matrix that we consider in (6) is of companion type, Cayley-Hamilton theorem gives

$$
A^{n}=-\sum_{m=0}^{n-1} a_{m} A^{m} .
$$

Therefore, for $m=n$ (96) simply gives

$$
\begin{aligned}
& \varepsilon^{(n)}-\sum_{l=1}^{n} K A^{l-1} B \varepsilon^{(n-l)}=f_{Z}^{n}(t)+f_{\varepsilon}^{n}(t)+f_{\tilde{X}}^{n}(t) \\
& -\sum_{m=0}^{n-1} a_{m}\left[(1+\dot{D})^{n} K e^{A D} A^{m} Z+K \int_{t-D}^{t} A^{m} e^{A(t-s)} B \varepsilon(s) d s\right]
\end{aligned}
$$

or again, using now (96) for $m$ ranging from 1 to $n-1$

$$
\begin{aligned}
& \varepsilon^{(n)}-\sum_{l=1}^{n} K A^{l-1} B \varepsilon^{(n-l)}=f_{Z}^{n}(t)+f_{\varepsilon}^{n}(t)+f_{\tilde{X}}^{n}(t) \\
& -\sum_{m=1}^{n-1} a_{m}(1+\dot{D})^{n-m}\left[\varepsilon^{(m)}-\sum_{l=1}^{m} K A^{l-1} B \varepsilon^{(m-l)}\right. \\
& \left.-f_{Z}^{m}(t)-f_{\varepsilon}^{m}(t)-f_{\tilde{X}}^{m}(t)-K \int_{t-D}^{t} A^{m} e^{A(t-s)} B \varepsilon(s) d s\right]
\end{aligned}
$$

$$
\begin{aligned}
& -\sum_{m=1}^{n-1} a_{m} K \int_{t-D}^{t} A^{m} e^{A(t-s)} B \varepsilon(s) d s \\
& -a_{0}\left[(1+\dot{D})^{n} K e^{A D} Z+K \int_{t-D}^{t} e^{A(t-s)} B \varepsilon(s) d s\right] .
\end{aligned}
$$

Besides, using the Leibniz formula

$$
(1+\dot{D})^{n-m}=1+\sum_{l=1}^{n-m}\left(\begin{array}{c}
n-m \\
l
\end{array}\right) \dot{D}^{l}
$$

and the expression of (94), one can define

$$
\begin{aligned}
& \pi_{1}\left(\dot{D}, \ldots, D^{(n)}, \varepsilon_{t}, \ldots, \varepsilon_{t}^{(n-1)}\right) \triangleq f_{Z}^{n}(t)+f_{\varepsilon}^{n}(t)+f_{\tilde{X}}^{n}(t) \\
& +\sum_{m=0}^{n-1} a_{m}(1+\dot{D})^{n-m}\left(f_{Z}^{m}(t)+f_{\varepsilon}^{m}(t)+f_{\tilde{X}}^{n}(t)\right) \\
& -\sum_{m=1}^{n-1} a_{m}\left[\sum_{l=1}^{n-m}\left(\begin{array}{c}
n-m \\
l
\end{array}\right) \dot{D}^{l}\right]\left[\varepsilon^{(m)}-\sum_{l=1}^{m} K A^{l-1} B \varepsilon^{(m-l)}\right. \\
& \left.-K \int_{t-D}^{t} A^{m} e^{A(t-s)} B \varepsilon(s) d s\right]-a_{0} \sum_{l=1}^{n}\left(\begin{array}{c}
n \\
l
\end{array}\right) \dot{D}^{l}[\varepsilon(t) \\
& \left.-K \int_{t-D}^{t} e^{A(t-s)} B \varepsilon(s) d s\right] .
\end{aligned}
$$

From there, one can obtain the dynamic (72) by observing that

$$
\begin{array}{r}
\sum_{l=1}^{n} K A^{l-1} B \varepsilon^{(n-l)}+\sum_{m=1}^{n-1} \sum_{l=1}^{m} a_{m} K A^{l-1} B \varepsilon^{(m-l)} \\
=-\sum_{l=1}^{n} b_{0} k_{n-l} \varepsilon^{(n-l)}
\end{array}
$$

which is proven in the next section and using Lemma 8 applied to the range $n$ to obtain the existence of a suitable function $\pi_{2}$. Further, from (109), using Lemma 7 and $9, \pi_{1}$ is a polynomial in the variables $\varepsilon_{t}, \ldots, \varepsilon_{t}^{(n-1)}, \dot{D}, \ldots, D^{(n)}, \tilde{X}$ and $1 / 1+\dot{D}$, at least quadratic in the variables $\varepsilon_{t}, \ldots, \varepsilon_{t}^{(n-1)}, \tilde{X}, \dot{D}, \ldots, D^{(n)}$.

\section{Proof of (110) Using Companion Matrix Properties}

One can reformulate the term under consideration in (110) as follows:

$$
\begin{array}{rl}
\sum_{l=1}^{n} & K A^{l-1} B \varepsilon^{(n-l)}+\sum_{m=1}^{n-1} \sum_{l=1}^{m} a_{m} K A^{l-1} B \varepsilon^{(m-l)} \\
= & \sum_{l=1}^{n} K A^{l-1} B \varepsilon^{(n-l)} \\
& +\sum_{p=2}^{n} \sum_{m=n-1-p}^{n-1} a_{m} K A^{p-n+m-1} B \varepsilon^{(n-p)} \\
= & K B \varepsilon^{(n-1)} \\
& +\sum_{l=2}^{n}\left[K A^{l-1} B+\sum_{m=n-1-l}^{n-1} a_{m} K A^{l-n+m-1} B\right] \varepsilon^{(n-l)} .
\end{array}
$$


To study the second term in (111), consider $m_{j}^{i}$ the $j^{\text {th }}$ coefficient of $A^{i} B$. As $A$ is a companion-type matrix, one gets

$$
m_{j}^{i}=\left\{\begin{array}{cl}
0 & \text { if } j \leq n-i-1 \\
m_{j+1}^{i-1} & \text { if } n-i \leq j \leq n-1 \\
-\sum_{l=n-i+1}^{n} a_{j-1} m_{j}^{i-1} & \text { if } j=n
\end{array}\right.
$$

and that

for $i \in \mathbb{N}, 1 \leq j \leq n$ and $1-j \leq p \leq \max \{i, n-j\}$

$$
m_{i}^{j}=m_{j+p}^{i-p} .
$$

Then, the coefficient of the $(n-p)^{t h}$ derivative can be rewritten as follows:

$$
\begin{aligned}
K A^{l-1} B+\sum_{m=n-1-p}^{n-1} a_{m} K A^{p-n+m-1} B \\
=K A^{l-1} B+\sum_{i=1}^{l-1} a_{n+i-l} K A^{i-1} B \\
=-k_{n-l} m_{n-l+1}^{l-1} \\
\quad-\sum_{j=n-l+2}^{n} k_{j-1}\left[m_{j}^{l-1}+\sum_{i=n-j+1}^{l-1} a_{n+i-l} m_{j}^{i-1}\right] .
\end{aligned}
$$

We now prove by induction that, for $l \geq 0$ and for $n-l+2 \leq$ $j \leq n, m_{j}^{l-1}=-\sum_{i=n-j+1}^{l-1} a_{n+i-l} m_{j}^{i-1}$.

For $l=2$ and $j=n$, the proposition is indeed true as $m_{j}^{l-1}=-a_{n-1} b_{0}$ and $-\sum_{i=n-j+1}^{l-1} a_{n+i-l} m_{j}^{i-1}=-a_{n-1} m_{n}^{0}$. Now, assume that the property is true for a given $l \geq 2$ and for all integer $j n-l+2 \leq j \leq n$. Consider $j$ such that $n-l+$ $2 \leq j \leq n-1$, then

$$
\begin{aligned}
\sum_{i=n-j+1}^{l} a_{n+i-l-1} m_{j}^{i-1} & =\sum_{i=n-j}^{l-1} a_{n+i-l} m_{j}^{i} \\
& =\sum_{i=n-j}^{l-1} a_{n+i-l} m_{j+1}^{i-1} \\
& =-m_{j+1}^{l-1}=-m_{j}^{l}
\end{aligned}
$$

using (112) and the induction assumption. This gives the desired result for $n-l+2 \leq j \leq n-1$. For $j=n$, one gets using successively (113) and (112)

$$
\begin{aligned}
\sum_{i=1}^{l} a_{n+i-l-1} m_{n}^{i-1} & =\sum_{i=1}^{l} a_{n+i-l-1} m_{i}^{n-1} \\
& =\sum_{i=n+1-l}^{n} a_{j-1} m_{j}^{l-1}=-m_{n}^{l}
\end{aligned}
$$

which gives the conclusion. Therefore, for $2 \leq l \leq n$

$$
\begin{aligned}
K A^{l-1} B+\sum_{m=n-1-p}^{n-1} a_{m} K A^{p-n+m-1} B & =-k_{n-l} m_{n-l+1}^{l-1} \\
& =-k_{n-l} m_{n}^{0}=-k_{n-l} b_{0}
\end{aligned}
$$

and $K B=-k_{n-1} b_{0}$. This concludes the proof of (110).

\section{ACKNOWLEDGMENT}

The authors are thankful to L. Praly for his very accurate and constructive criticisms on an early version of this paper.

\section{REFERENCES}

[1] Z. Artstein, "Linear systems with delayed controls: A reduction," IEEE Trans. Autom. Control, vol. 27, no. 4, pp. 869-879, 1982.

[2] B. Balachandran, T. Kalmar-Nagy, and D. E. Gilsinn, Delay Differential Equations: Recent Advances and New Directions. Berlin, Germany: Springer Verlag, 2009.

[3] N. Bekiaris-Liberis and M. Krstic, "Compensation of time-varying input and state delays for nonlinear systems," J. Dyn. Syst., Meas. Control, vol. 134, 2012, Paper 011009.

[4] N. Bekiaris-Liberis and M. Krstic, "Compensation of state-dependent input delay for nonlinear systems," IEEE Trans. Autom. Control, vol. 58, pp. 275-289, 2013.

[5] N. Bekiaris-Liberis and M. Krstic, "Nonlinear control under delays that depend on delayed states," Eur. J. Control, Special Issue ECC'13, vol. 19, pp. 389-398, 2013.

[6] N. Bekiaris-Liberis and M. Krstic, "Robustness of nonlinear predictor feedback laws to time-and state-dependent delay perturbations," Automatica, vol. 49, pp. 1576-1590, 2013.

[7] D. Bresch-Pietri, "Robust control of variable time-delay systems. Theoretical contributions and applications to engine control," $\mathrm{PhD}$ thesis, MINES ParisTech, Paris, France, 2012.

[8] D. Bresch-Pietri, J. Chauvin, and N. Petit, "Adaptive control scheme for uncertain time-delay systems," Automatica, vol. 48, pp. 1536-1552, 2012.

[9] D. Bresch-Pietri, J. Chauvin, and N. Petit, "Invoking Halanay inequality to conclude on closed-loop stability of a process with input-varying delay," in Proc. 10th IFAC Workshop Time Delay Syst., 2012, pp. 266-271.

[10] D. Bresch-Pietri, J. Chauvin, and N. Petit, "Sufficient condition for prediction-based stabilization of linear system subject to input-dependent input-delay," in Proc. Amer. Control Conf., 2013, pp. 144-151.

[11] D. Bresch-Pietri, T. Leroy, J. Chauvin, and N. Petit, "Practical delay modeling of externally recirculated burned gas fraction for spark-ignited engines," in Proc. 11th Workshop Time-Delay Syst., 2013, pp. 232-237.

[12] M. Chébre, Y. Creff, and N. Petit, "Feedback control and optimization for the production of commercial fuels by blending," J. Process Control, vol. 20, no. 4, pp. 441-451, 2010.

[13] C. Depcik and D. Assanis, "One-dimensional automotive catalyst modeling," Progress Energy Combustion Sci., vol. 31, no. 4, pp. 308-369, 2005.

[14] K. Gu and S. I. Niculescu, "Survey on recent results in the stability and control of time-delay systems," J. Dyn. Syst., Meas., Control, vol. 125, p. $158,2003$.

[15] A. Halanay, Differential Equations: Stability, Oscillations, Time Lags, vol. 23. New York: Academic Press, 1966.

[16] J. Harmand and D. Dochain, "The optimal design of two interconnected (bio) chemical reactors revisited," Comp. Chem. Eng., vol. 30, no. 1, pp. 70-82, 2005.

[17] J. P. Hathout, M. Fleifil, A. M. Annaswamy, and A. F. Ghoniem, "Active control using fuel-injection of time-delay induced combustion instability," AIAA J. Propulsion Power, vol. 18, pp. 390-399, 2002.

[18] A. Ivanov, E. Liz, and S. Trofimchuk, "Halanay inequality, yorke 3/2 stability criterion, differential equations with maxima," Tohoku Math. J., vol. 54, no. 2, pp. 277-295, 2002.

[19] M. Jankovic, "Recursive predictor design for linear systems with time delay," in Proc. Amer. Control Conf., 2008, pp. 4904-4909.

[20] I. Karafyllis and M. Krstic, "Delay-robustness of linear predictor feedback without restriction on delay rate," Automatica, vol. 49, pp. 1761-1767, 2013.

[21] M. Krstic, Boundary Control of PDEs: A Course on Backstepping Designs. SIAM, Philadelphia, PA, USA, 2008.

[22] M. Krstic, Delay Compensation for Nonlinear, Adaptive, PDE Systems. Boston, MA: Birkhauser, 2009

[23] B. Liu, W. Lu, and T. Chen, "Generalized halanay inequalities and their applications to neural networks with unbounded time-varying delays," IEEE Trans. Neural Networks, vol. 22, no. 9, pp. 1508-1513, 2011.

[24] A. Manitius and A. Olbrot, "Finite spectrum assignment problem for systems with delays," IEEE Trans. Autom. Control, vol. 24, no. 4, pp. 541-552, 1979.

[25] W. Michiels and S. I. Niculescu, Stability and Stabilization of Time-Delay Systems. SIAM, Philadelphia, PA, USA, 2007.

[26] Y. S. Moon, P. G. Park, and W. H. Kwon, "Robust stabilization of uncertain input-delayed systems using reduction method," Automatica, vol. 37, no. 2, pp. 307-312, 2001. 
[27] M. S. Natanzon, Combustion instability, vol. 222. Reston, VA: AIAA, 1999.

[28] M. T. Nihtila, "Finite pole assignment for systems with time-varying input delays," in Proc. 30th IEEE Conf. Decision Control, 1991, pp. 927-928.

[29] R. H. Perry, D. W. Green, and J. O. Maloney, Perry's Chemical Engineers' Handbook, vol. 7. New York: McGraw-Hill, 1984.

[30] N. Petit, Y. Creff, and P. Rouchon, "Motion planning for two classes of nonlinear systems with delays depending on the control," in Proc. 37th IEEE Conf. Decision Control, 1998, pp. 1007-1011.

[31] J.-P. Richard, "Time-delay systems: An overview of some recent advances and open problems," Automatica, vol. 39, no. 10, pp. 1667-1694, 2003.

[32] M. Sbarciog, R. De Keyser, S. Cristea, and C. De Prada, "Nonlinear predictive control of processes with variable time delay. A temperature control case study," in Proc. IEEE Int. Conf. Control Appl., 2008, pp. 1001-1006.

[33] O. J. M. Smith, "A controller to overcome dead time," ISA J., vol. 6, no. 2, pp. 28-33, 1959.

[34] W. Wansheng, "A generalized halanay inequality for stability of nonlinear neutral functional differential equations," J. Inequalities Appl., 2010:475019.

[35] E. Witrant, "Stabilisation des Systèmes commandés par réseaux," Ph.D. dissertation, Laboratoire d'Automatique de Grenoble, Grenoble, France, 2005.

[36] Y. Yildiz, A. Annaswamy, D. Yanakiev, and I. Kolmanovsky, "Spark ignition engine fuel-to-air ratio control: An adaptive control approach," Control Eng. Practice, vol. 18, no. 12, pp. 1369-1378, 2010.

[37] D. Yue and Q. L. Han, "Delayed feedback control of uncertain systems with time-varying input delay," Automatica, vol. 41, no. 2, pp. 233-240, 2005.

[38] K. Zenger and A. J. Niemi, "Modelling and control of a class of timevarying continuous flow processes," J. Process Control, vol. 19, no. 9, pp. 1511-1518, 2009.

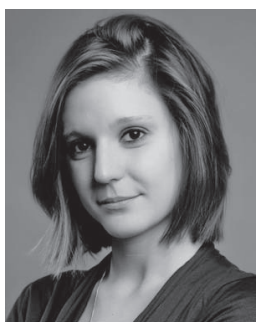

Delphine Bresch-Pietri received the M.S. degree in science and executive engineering and the $\mathrm{Ph} . \mathrm{D}$. degree in mathematics and control from MINES ParisTech, Paris, France, in 2009 and 2012, respectively.

From 2009 to 2012, she worked jointly between the Centre Automatique et Systèmes at MINES ParisTech and IFP Energies nouvelles. She was a PostDoctoral Associate in Mechanical Engineering at the Massachusetts Institute of Technology, Cambridge, in 2013. Since 2014, she has been a Chargée de Recherche at CNRS, GIPSA-lab, Grenoble, France. She is the coauthor of several patents in the field of engine control. Her research interests include engine control, theory and applications of time-delay systems, boundary control of partial differential equations, adaptive design and safety control for semi-autonomous agents.

Dr. Bresch-Pietri received the ParisTech Best Ph.D. Award in 2013.

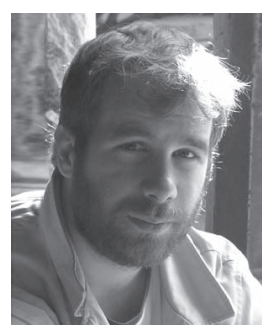

Jonathan Chauvin was born in La Roche-sur-Yon, France, in 1981. He received the M.S. degree and the $\mathrm{Ph} . \mathrm{D}$. degree in mathematics and control from the Ecole des Mines de Paris, Paris, France, in 2003 and 2006, respectively.

$\mathrm{He}$ is now innovation project leader in the control, signal and system department at the IFP Energies nouvelles. He is the coauthor of several patents in the field of engine and renewable energy system control. His fields of interest include the theory and applications of dynamical systems, engine control, renewable energy systems and in periodic systems.

Dr. Chauvin received the Paristech Best Ph.D. Award in 2007.

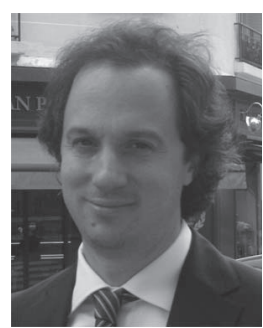

Nicolas Petit was born in Paris, France, in 1972. He received the M.S. degree from Ecole Polytechnique, Palaiseau, France, in 1995, the Ph.D. degree in mathematics and control from MINES ParisTech, Paris, France, in 2000, and the Habilation á Diriger des Recherches from Université Paris 6, Paris, France, in 2007

$\mathrm{He}$ is Professor and Head of the Centre $\mathrm{Au}-$ tomatique et Systèmes, MINES ParisTech. He has developed the controllers of several industrial chemical reactors, and the patented softwares ANAMEL 4 and 5, currently used for closed-loop control of blending devices in numerous refineries at the TOTAL company. $\mathrm{He}$ is the co-founder and was Scientific Director of Sysnav from 2008 to 2013 He has been a board member of Wandercraft since 2013. He has served as an Associate Editor for Automatica since 2006 and has been an Associate Editor and Subject Editor for the Journal of Process Control since 2012 and 2014, respectively. His research interests include distributed parameter systems, constrained optimal trajectory generation, delay systems, and observer design. On the application side, he is active in industrial process control, control of multiphasis flow, internal combustion engine control, and inertial navigation.

Dr. Petit received the "Best Paper Prize" from the Journal of Process Control, for the periods 2002-2005 and 2008-2011, respectively. 